\title{
Field study of the dynamics and modelling of subgrid-scale turbulence in a stable atmospheric surface layer over a glacier
}

\author{
ELIE BOU-ZEID ${ }^{1} \uparrow$, CHAD HIGGINS ${ }^{2}$, \\ HENDRIK HUWALD ${ }^{2}$, CHARLES MENEVEA U \\ AND MARC B. PARLANGE \\ ${ }^{1}$ Department of Civil and Environmental Engineering, Princeton University, E414 EQuad, \\ Princeton, NJ 08544, USA \\ ${ }^{2}$ School of Architecture, Civil and Environmental Engineering, École Polytechnique Fédérale de \\ Lausanne-EPFL, Lausanne, Switzerland \\ ${ }^{3}$ Department of Mechanical Engineering, Johns Hopkins University, Baltimore, MD 21218-2680, USA
}

(Received 19 August 2009; revised 26 July 2010; accepted 26 July 2010)

A field experiment - the Snow Horizontal Array Turbulence Study (SnoHATS) has been performed over an extensive glacier in Switzerland in order to study smallscale turbulence in the stable atmospheric surface layer, and to investigate the role, dynamics and modelling of the subgrid scales (SGSs) in the context of large-eddy simulations. The a priori data analysis aims at comparing the role and behaviour of the SGSs under stable conditions with previous studies under neutral or unstable conditions. It is found that the SGSs in a stable surface layer remain an important sink of temperature variance and turbulent kinetic energy from the resolved scales and carry a significant portion of the fluxes when the filter scale is larger than the distance to the wall. The fraction of SGS fluxes (out of the total fluxes) is found to be independent of stability. In addition, the stress-strain alignment is similar to the alignment under neutral and unstable conditions. The model coefficients vary considerably with stability but in a manner consistent with previous findings, which also showed that scale-dependent dynamic models can capture this variation. Furthermore, the variation of the coefficients for both momentum and heat SGS fluxes can be shown to be better explained by stability parameters based on vertical gradients, rather than vertical fluxes. These findings suggest that small-scale turbulence dynamics and SGS modelling under stable conditions share many important properties with neutral and convective conditions, and that a unified approach is thus possible. This paper concludes with a discussion of some other challenges for stable boundary-layer simulations that are not encountered in the neutral or unstable cases.

Key words: atmospheric flows, stratified turbulence, turbulent boundary layers, turbulence modelling

\section{Introduction}

Stably stratified atmospheric flows are usually characterized by weak and highly anisotropic turbulence, gravity waves, low-level jets, Kelvin-Helmholtz instabilities 
and meandering motions that are not observed in neutral or unstable atmospheric flows (Mahrt 1998; Chimonas 1999; Derbyshire 1999; Armenio \& Sarkar 2002; Cheng \& Brutsaert 2005; Cheng, Parlange \& Brutsaert 2005). These features complicate both modelling and measurements under statically stable conditions. On the experimental side, the usual flux-gradient or flux-variance relations that are based on classic turbulence similarity theories for the lower atmosphere, such as the Monin-Obukhov similarity theory (MOST), are more challenging to apply (Pahlow, Parlange \& Porte-Agel 2001). MOST assumes that turbulence, generated near the surface, is the overwhelmingly dominant flow characteristic; this is not always valid under stable conditions. In addition, a constant-flux surface layer may not be well established and hence flux measurements at a given height might not equal surface fluxes as is the case under neutral and statically unstable conditions (we use the term 'flux' in this paper to refer to both momentum fluxes, i.e. stresses and heat fluxes). On the modelling side, the main problem is that classic turbulence parameterizations have often been found to be inadequate under stable conditions, especially under strong stability (see review in Mahrt 1998).

Most of the studies and reviews that point to difficulties in modelling the stable atmospheric boundary layer (ABL) examine very coarse models (such as weather prediction models), where the whole ABL is parameterized, or Reynolds-averaged Navier-Stokes (RANS) models, where all the scales of turbulence are parameterized. These findings about the challenges in modelling the stable ABL have lead researchers to expect similar difficulties when using the large-eddy simulation (LES) technique, where only scales smaller than the computational grid (or filter size) are parameterized. However, recent evidence suggests that LES yields better results under stable conditions (Beare et al. 2006; Kleissl et al. 2006; Stoll \& Porte-Agel 2008) and can even capture physical features of the diurnal cycle such as nocturnal jet dynamics (Kosovic \& Curry 2000; Beare \& Macvean 2004; Beare et al. 2006; Kumar et al. 2006). This is not an entirely surprising finding since in LES only the smallest scales of turbulence are parameterized; the large scales are explicitly modelled and can dynamically adjust to the effect of stability. However, the good performance of LES in specific test cases under weakly-stable conditions, while very encouraging, is not a sufficient assurance that the technique will perform well in other test cases, especially under strongly-stable conditions (Mahrt 1998). For example, Kumar et al. (2006) found that when the stability factor $\Delta / L_{M O}$ (where $\Delta$ is the grid filter scale and $L_{M O}$ is the Obukhov length scale) is increased beyond 4, numerical instabilities developed, suggesting that such strongly stable conditions might be more challenging for either the subgrid-scale (SGS) models or the numerical discretization schemes.

Further testing of the LES technique, and of the related SGS turbulence models, is needed in view of the promise this computational technique offers for understanding the effects of stratification and stability in geophysical and engineering flows. LES-based studies are expected to lead to a better understanding of complex interactions involving waves and turbulence, instabilities, heterogeneous and complex geometries and transient forcing effects. This will then lead to improved sensing and modelling of stable boundary-layer flow and transport and to enhanced ABL parameterizations in weather and climate models. This last goal is very critical; the $\mathrm{ABL}$ is the link through which all atmosphere-surface interactions occur: moisture and heat are injected into the free troposphere through the $\mathrm{ABL}$, while for momentum and turbulent kinetic energy (TKE), the ABL acts as the main sink. Not surprisingly, weather (Beljaars 1995; Han, Ueda \& An 2008) and climate (Koster et al. 2006; Guo et al. 2006) models continue to show significant sensitivity to ABL parameterizations. 
The main problem with large-scale models is that current ABL parameterizations for stable conditions tend to underestimate the level of TKE, and consequently heat fluxes, during night time. This leads to unphysical surface cooling, which then damps the turbulence further leading to 'run away' surface cooling (Steeneveld, Van de Wiel \& Holtslag 2006). This positive feedback could drive the model into a state of radiative balance with the turbulence being completely damped. This misrepresentation of turbulence and mixing intensity in the stable ABL prevents the correct modelling of night-time pollutant dispersion, fog and road frost formation, and polar boundary layers and climates (see King, Connolley \& Derbyshire 2001 on the last topic).

About a decade ago Chimonas (1999) noted that 'LES provide the best hope for synthesizing a realistic model' of the stable boundary layer. Mahrt (1998) and Derbyshire (1999) noted the lack of experimental data that can be used to test SGS models for stable ABLs. Since then, the gap has been partially bridged with several studies looking at the performance of several SGS models under stable conditions and investigating the effect of stability on the value of optimal SGS model coefficients using either direct numerical simulation (DNS) data (Dubrulle et al. 2002) or, more frequently, field experimental data (Tong, Wyngaard \& Brasseur 1999; Porte-Agel et al. 2001a, b; Sullivan et al. 2003; Kleissl, Meneveau \& Parlange 2003; Horst et al. 2004; Kleissl, Parlange \& Meneveau 2004; Chamecki, Meneveau \& Parlange 2007; Bou-Zeid et al. 2008; Higgins, Meneveau \& Parlange 2009).

The goal of this paper is to answer several remaining open questions related to the effect of stability on the role and dynamics of the small, unresolved scales. What is the main function of the SGSs under stable conditions and is it fundamentally different from neutral or unstable conditions? Do SGS fluxes and dissipations (of TKE and scalar variance) significantly increase in importance (as a fraction of the total fluxes and dissipations) as stability increases? Are there inherent difficulties in modelling SGS turbulence under stable and very stable conditions similar to the difficulties faced when using RANS models? Are model coefficients more challenging to estimate under stable conditions? The answers to the first two questions are independent of what SGS model is being considered, while the answers to the last two are related to the choice of SGS model. When studying challenges in modelling SGS turbulence under stable conditions (third question), we consider a generic eddy-viscosity model as an example. Eddy-viscosity models remain the most widely used in LES of geophysical and engineering flows and they encompass a wide range of models that essentially differ in the way the SGS eddy viscosity is computed such as the SmagorinskyLilly model (Smagorinsky 1963; Lilly 1967), the Wong-Lilly model (Wong \& Lilly 1994), structure function models (e.g. Metais \& Herring 1989; Cui et al. 2004) and numerous models based on SGS kinetic energy (e.g. Lilly 1967; Stevens, Moeng \& Sullivan 1999). As such, a large body of results based on this model exist for the neutral and unstable ABLs and using it allows us to address the main objective of this paper, which is to investigate the specific physics and modelling challenges that arise under stable conditions. The fourth question, dealing with model coefficients, is investigated in the context of the Smagorinsky model.

\section{Subgrid-scale fluxes}

In LES, the scales of motion that are larger than the grid (or filter) scale are explicitly resolved, i.e. their prognostic equations, the filtered Navier-Stokes equations, are solved numerically. These equations are obtained by applying a filtering operation to the full Navier-Stokes equations to remove the contribution of all the scales that 
are smaller than the grid size, the so-called SGSs. Due to the strong nonlinear scale interactions that characterize turbulence, the SGSs have a substantial effect on the resolved scales, which has to be parameterized to obtain an accurate solution for the large scales. This effect appears in the filtered Navier-Stokes equations as the divergence of the SGS stress

$$
\sigma_{i j}=\widetilde{u_{i} u_{j}}-\widetilde{u_{i}} \widetilde{u_{j}}
$$

where $u_{1}, u_{2}$ and $u_{3}$ are the streamwise, cross-stream and vertical velocity components, respectively (alternatively referred to as $u, v$ and $w$ later in this paper), and the tilde $(\widetilde{u})$ represents the filtering operation and the filtered quantities. Later in this paper, we will use the double prime $\left(u^{\prime \prime}\right)$ to denote the SGS quantities, while Reynolds averaging will be denoted by an overbar $(\bar{u})$ or brackets $(\langle u\rangle)$ and the turbulent fluctuating quantities will be denoted by a single prime $u^{\prime}$, hence $u=\widetilde{u}+u^{\prime \prime}=\bar{u}+u^{\prime}$. To simplify the SGS modelling, the isotropic component of the SGS stress tensor is usually combined with the pressure term and the SGS model only parameterizes the effects of the anisotropic (deviatoric) part

$$
\tau_{i j}=\sigma_{i j}-\frac{1}{3} \sigma_{k k} \delta_{i j}
$$

SGS modelling is also needed when solving the filtered heat transport equation, where the effect of the unresolved scales appears as the divergence of the SGS heat flux

$$
q_{i}=\widetilde{u_{i} \theta}-\widetilde{u_{i}} \widetilde{\theta}
$$

where $\theta$ is the potential temperature (detailed definition of $\theta$ is given later in this paper).

The results of LES are quite sensitive to the SGS model, especially in flows or regions of the flow where the SGS contribution is important, such as in the vicinity of solid boundaries (the atmospheric surface layer for example), or in flows where the turbulence markedly departs from the homogenous isotropic state (Canuto \& Minotti 1993; Kosovic 1997; Meneveau \& Katz 2000; Porte-Agel, Meneveau \& Parlange 2000a; Gullbrand \& Chow 2003; Sullivan et al. 2003; Bou-Zeid, Meneveau \& Parlange 2005).

A large variety of SGS models have been developed and tested for various flows. Model testing is performed either a posteriori, i.e. results from an LES are compared to results for the same flow obtained through experimental measurements or DNS, or a priori, i.e. highly-resolved turbulent fields from experimental observations or DNS are filtered to split the turbulence into resolved and SGS parts (Meneveau 1994; Tong et al. 1999), which are then used to study SGS physics and test the ability of models to reproduce the measured SGS parameters. As discussed previously, the goal of this paper is to study the specific characteristics of the small scales in statically stable wall-bounded flows and to contrast them to statically neutral and unstable flows. Since the most widely used model for the SGSs remains the Smagorinsky model or variants of that model (based on the eddy-viscosity and mixing-length assumptions, see Meneveau \& Katz 2000), we use this modelling approach as an example in this paper, where needed. The model relates the SGS stress to the resolved strain rate tensor $\widetilde{S}_{i j}=0.5\left(\partial \widetilde{u}_{i} / \partial x_{j}+\partial \widetilde{u}_{j} / \partial x_{i}\right)$ via an SGS eddy viscosity, $v_{S G S}$, formulated as a length scale $\left(c_{s} \Delta\right)$ multiplied by a velocity scale $\left(c_{s} \Delta|\widetilde{S}|\right)$

$$
\tau_{i j}^{\text {model }}=-2 v_{S G S} \widetilde{S}_{i j}=-2\left(c_{s} \Delta\right)^{2}|\widetilde{S}| \widetilde{S}_{i j}
$$


(a)

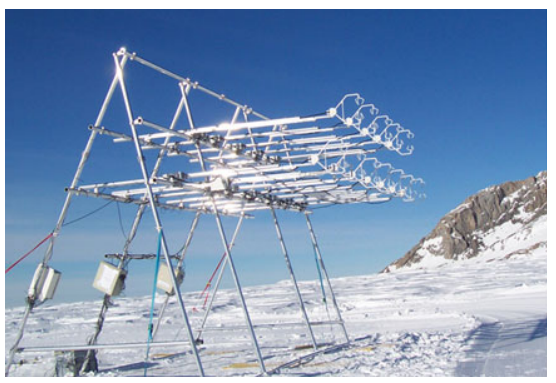

(b)

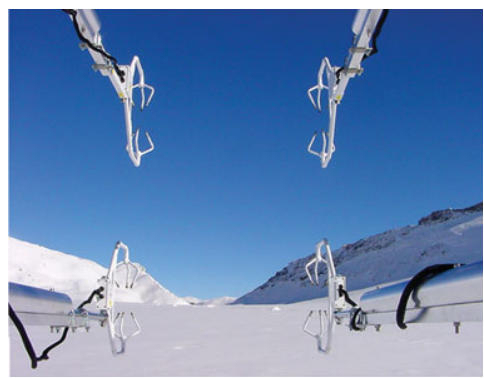

Figure 1. SnoHATS: side view of the 12 sonics array $(a)$ and the upwind fetch of $1.5 \mathrm{~km}(b)$.

where $|\widetilde{S}|=\left(2 \widetilde{S}_{i j} \widetilde{S}_{i j}\right)^{1 / 2}$ is the magnitude of resolved strain-rate tensor, $c_{s}$ is the Smagorinsky coefficient and $\Delta$ is the filter scale. Similarly, the SGS heat flux is related to the potential temperature gradient via an eddy-diffusivity, $k_{S G S}$, using the SGS turbulent Prandtl number, $P r_{S G S}$ :

$$
q_{i}^{\text {model }}=-k_{S G S} \frac{\partial \widetilde{\theta}}{\partial x_{i}}=-\operatorname{Pr}_{S G S}^{-1}\left(c_{S} \Delta\right)^{2}|\widetilde{S}| \frac{\partial \widetilde{\theta}}{\partial x_{i}} .
$$

\section{Experimental set-up}

To help answer the questions raised in $\S 1$, a field study was performed over the extensive 'Plaine-Morte' glacier in the Swiss Alps $\left(7.5178^{\circ} \mathrm{E}, 46.3863^{\circ} \mathrm{N}, 2750 \mathrm{~m}\right.$ elevation) from 2 February to 19 April 2006. The snow cover provided stable stratification of the flow over long periods, especially under clear-sky conditions (see study of cloudiness effect on stability in Mirocha, Kosovic \& Curry 2005). The goal of the experiment was to allow the computation of the relevant SGS variables introduced before, $\tau_{i j}, \widetilde{S}_{i j}, q_{i}$ and $\partial \widetilde{\theta} / \partial x_{i}$ which would then also yield the mean rates of SGS dissipations of TKE, $-\left\langle\tau_{i j} \widetilde{S}_{i j}\right\rangle$, and of potential temperature variance, $-\left\langle q_{i} \partial \widetilde{\theta} / \partial x_{i}\right\rangle$. Therefore, two vertically-separated horizontal arrays with a total of 12 sonic anemometers (three-dimensional, Campbell Scientific CSAT3) were deployed (figure 1) to allow two-dimensional filtering and computation of the full three-dimensional gradients; the experiment was hence called the Snow Horizontal Array Turbulence Study (SnoHATS). Supporting measurements at the site included mean wind speed and direction (Vector Instruments A100R and W200P, respectively), temperature and relative humidity of the air at several heights (Rotronic MP103A), the four radiation components (Kipp and Zonen CM21 for shortwave up and down and CG4 for longwave up and down), snow elevation (Campbell Scientific SR50), snowsurface temperature (Apogee Instruments IRTS-P infrared thermocouple sensor) and high-frequency water vapour concentration (3 Campbell Scientific KH20 Krypton Hygrometers) for eddy-covariance measurement of evaporation.

Due to snow accumulation at the surface, the height of the two arrays above snow level varied between 2.82 and $0.62 \mathrm{~m}$. This variation is very useful since it allows the effect of the height above the surface to be studied. Data analysis was restricted to wind directions of $\pm 60^{\circ}$ relative to the streamwise sonic axis (corresponding to easterly winds); this ensures that the wind is blowing over a long fetch of flat snow (minimum of $1500 \mathrm{~m}$, to keep the instruments in the internal equilibrium layer: Brutsaert 1998; 
Bou-Zeid, Meneveau \& Parlange 2004; Bou-Zeid, Parlange \& Meneveau 2007) and that the flow is not disturbed by the presence of the support structure. In addition, easterly winds at the field site are typically dry and associated with clear skies, nice weather and no precipitation; this ensures optimal sensor operating conditions (sonic data are not reliable in rainy or snowy conditions). Drainage flows, waves and other similar stable flow features could very well be present in our measurement; they are an integral part of the complexity of stable ABLs and an SGS model should still perform well regardless of the generating mechanisms and dynamics of turbulence especially as, when performing LES of SABLs, one could, and often would like to, generate many of these realistic SABL features in the simulation. As such, we intentionally present most of our findings without any pre-filtering that would separate the turbulent and non-turbulent modes, since we intend to study realistic SGS physics and test SGS models under non-ideal, maybe challenging, conditions. However, since we are also interested in understanding when and how these features interact with the small turbulent (subgrid) scales, and how this interaction can be represented in an SGS model, we have tested the effects of pre-filtering large-scale modes using both a Gaussian high-pass filter and a multi-resolution decomposition (MRD) filter (Vickers \& Mahrt 2003; Nakamura \& Mahrt 2005) with filter sizes of 100-180 s. We report on the interesting findings of these tests in $\$ 11$.

After data periods with unfavourable wind angles or other data availability/quality problems (snow covered sonics, power outages,...) were removed, the equivalent of about 15 (non-continuous) days of data was available for analysis. The $20 \mathrm{~Hz}$ raw sonic data were collected on three Campbell Scientific CR5000 data loggers in a '1 master +2 slaves' configuration (the master data logger triggers the other 2 loggers and the measurements of all 12 sonics to ensure synchronization). The CSAT3 sonic performs several QA/QC tests in real time and automatically flags each record, where a problem is detected; the instantaneous data record was not used if any flag was 'active' for any of the 12 sonics. Pre-processing and data conditioning were performed afterwards and included axis rotation to correct the yaw and pitch misalignments of the sonics and linear detrending. Following previous similar studies (e.g. Porte-Agel et al. 2001b; Horst et al. 2004; Kleissl et al. 2003, 2004), roll correction was not deemed necessary and was hence not performed.

All instruments were tested and intercompared in the laboratory before deployment to ensure good calibration. In the laboratory, under zero wind conditions, most of the sonics had errors within the manufacturer's specification for offset $\left(0.04 \mathrm{~m} \mathrm{~s}^{-1}\right)$; three sonics had one individual component each exceeding the specified offset with the largest being the offset of the streamwise component of Sonic no. $12\left(0.16 \mathrm{~m} \mathrm{~s}^{-1}\right)$. Since these offsets are measured in the laboratory, we could correct them during the data analysis phase. This was done but, as expected, the effect on the results of this paper was not significant due to the filtering operations which average the data over five sonics, as explained later. The standard deviation of the mean temperature readings of the sonics in the laboratory was $0.41^{\circ} \mathrm{C}$; however, in the field, the sonics were arranged such that the different groups that will be filtered (averaged) together had almost the same combined mean temperature in the laboratory tests (difference in means of the four groups less than $0.04{ }^{\circ} \mathrm{C}$ ); no explicit correction for the temperature offsets was hence performed.

The sonics measure the so-called 'sonic temperature' $T_{s}=T(1+0.51 q)$, where $T$ is the temperature $(\mathrm{K})$ and $q$ is the specific humidity (mass of water vapour per unit mass of moist air). For our analysis we need the potential temperature, $\theta$, which is the temperature of the air parcel when brought adiabatically to a reference 
(a)
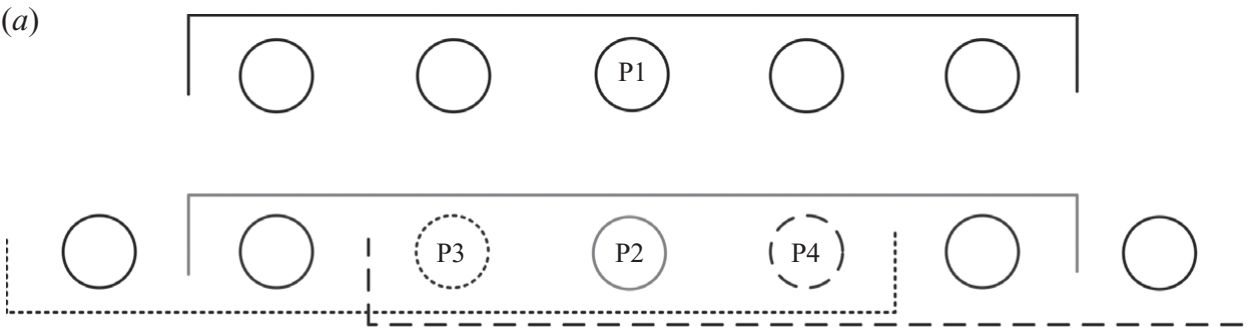

(b)

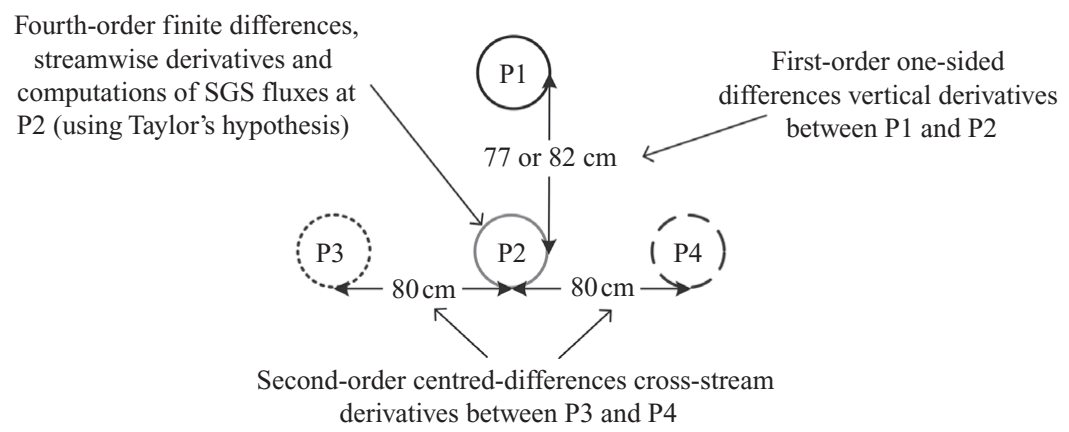

FIGURE 2. Filtering over groups of five sonics $(a)$ and computation of derivatives and SGS fluxes $(b)$.

pressure of $1000 \mathrm{hPa}$ and the virtual potential temperature $\theta_{v}=\theta(1+0.61 q)$, which is the potential temperature the air parcel will have when dried-up (at a constant pressure of $1000 \mathrm{hPa}$ ) and heated to regain its original moist-air density. The virtual potential temperature is frequently used in studies of atmospheric flows since it lumps the effects of variations in temperature, humidity and elevation (pressure) on air density. Since high frequency humidity measurements were not available for each sonic, we compute the instantaneous temperature using the mean specific humidity measurements from $T=T_{s} /(1+0.51\langle q\rangle)$; this has virtually no effect on the results due to the very low humidity of the air at the low temperatures over the glacier. The temperature readings are then converted to potential temperature following $\theta=T\left(p_{0} / p\right)^{R / c_{p}}=T(1000 / 720)^{287.04 / 1004.76}$, where $p=720 \mathrm{hPa}$ is the average pressure during the experiment (relative variations in $p$ are very small and can be neglected). Finally, the virtually potential temperature is computed using mean specific humidity $\theta_{v}=\theta(1+0.61\langle q\rangle)$.

\section{Computation of gradients, fluxes and dissipations}

The filtering operation denoted by the tilde in (2.1)-(2.5) was performed in two dimensions (see Higgins, Meneveau \& Parlange 2007 for comparison with threedimensional filters). A box filter was applied in the cross-stream direction and a Gaussian filter of the same effective size, $\Delta$, was applied in the streamwise direction by invoking Taylor's hypothesis to convert time series to streamwise spatial series. The filtering operation is applied separately to four groups of five sonics each, as depicted in figure 2; this effectively results in four data points, denoted P1 to P4 in the figure, 
where filtered variables are available. The vertical gradients are then computed from P1 and P2 (using first-order one-sided finite differences yielding the derivative values at P2). The vertical distance between points $\mathrm{P} 1$ and $\mathrm{P} 2$ is $\mathrm{d} z=0.77 \mathrm{~m}$ for the data before March 15 and $\mathrm{d} z=0.82 \mathrm{~m}$ after March 15, when the structure had to be lifted and reassembled due to snow accumulation at the surface. The cross-stream gradients are computed from P3 and P4 (using second-order centred differences around point $\mathrm{P} 2$ with $\mathrm{d} y=0.8 \mathrm{~m})$. Streamwise gradients are also computed from the data at P2 using Taylor's hypothesis (to convert time series to streamwise spatial series) and a forth-order centred-differences scheme using $\mathrm{d} x$ equal to $\mathrm{d} y$ (as recommended in Kleissl et al. 2003). Hence, all derivates are obtained at point P2 and the needed SGS fluxes, $\tau_{i j}$ and $q_{i}$, are also computed using the filtered data from the group of five sonics centred at P2.

For most computations, some averaging is required; in this paper, the averaging is performed in time over $30 \mathrm{~min}$ data records. The duration of the time averaging is selected as a compromise between short periods that exclude the effects of the meanflow unsteadiness resulting from synoptic forcing variability and the diurnal cycle, and longer periods that ensure convergence of the turbulence statistics (sampling enough realizations of the large eddies to compute the mean and higher-order moments; see discussion of convergence errors and averaging times in Wyngaard 1992; Kunkel \& Marusic 2006). In addition, we tested several averaging periods (15, 30 and 45 min) and observed no significant impacts on the deduced trends, apart from the expected increase in scatter associated with shorter averaging times.

Once the quantities of interest were obtained for each period, we imposed further data quality control tests including: (i) Taylor's hypotheses validity condition $\sigma_{u}<0.5\langle u\rangle$ (Willis \& Deardorff 1976), (ii) the standard deviation of the wind angles reported by the 12 sonics for each period is less than $5^{\circ}$, (iii) the tilt angles computed and used to perform the pitch correction do not exceed $2^{\circ}$, (iv) the standard deviation of friction velocities measured by the 12 sonics is smaller than 0.25 times the mean friction velocity for that period. These tests are designed to exclude any records with obvious problems in the data mainly by comparing the measurements of the 12 sonics. However, we note that tests showed that the overall trends are minimally sensitive to these limits (which mainly affected the scatter) and most records had agreements between the sonics and Taylor's hypothesis criteria that are much better than these ad hoc limits. After all these quality tests are done, the equivalent of about 6.2 days of data is left for analysis. Additional data quality checks were also done, following the recommendations in Vickers \& Mahrt (1997): (i) we have verified that for all the records we analyse, the variances of the temperature and velocities are never smaller than 10 times the sonic's manufacturer specified resolution, (ii) low pass filtering of the data with a filter scale of $0.15 \mathrm{~s}$ (corresponding to three data points) was tested to remove the spikes and no significant impact on the results was observed from such filtering (data presented here are without low pass filtering), (iii) linear detrending was performed rather than the exclusion of records that display large unsteadiness; this was found not to introduce undesirable artifacts or errors, (iv) the many visual inspections of the data records showed no 'dropouts'; furthermore, the Campbell Scientific sonic anemometers used here have several real-time QA/QC checks that flag any records where the sonic is not performing optimally and those records are not used in the analysis and (v) finally, flux-loss correction due to low sensor resolution has not been implemented, since the corrections require models for the covariance. These covariance models might be uncertain under strongly stable conditions and extrapolation from the resolved scales might also be uncertain. 


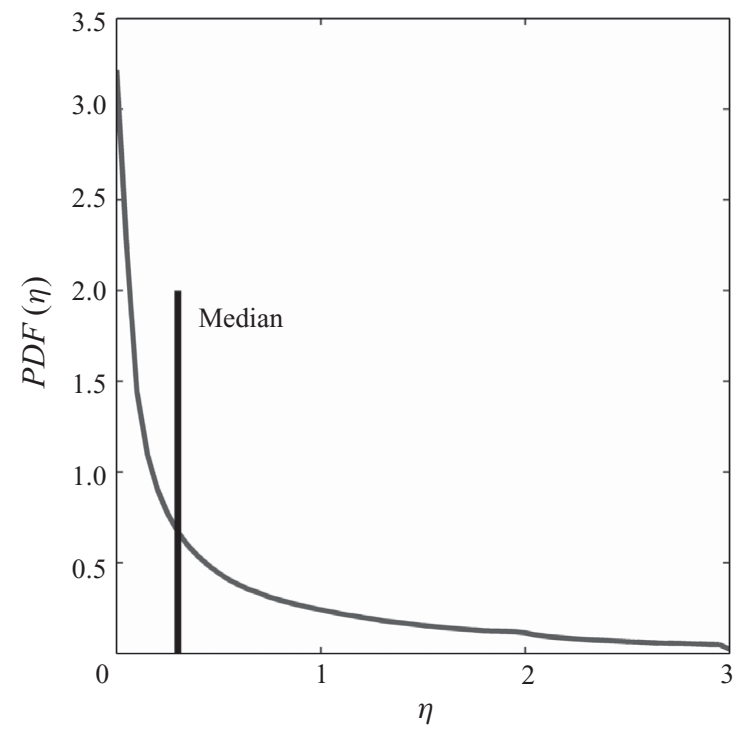

FIGURE 3. Probability density function of the normalized divergence of the measured velocities; accurate and precise measurements and gradient computations should yield $\eta=0$.

\section{Quality of the computed derivatives}

Following Zhang, Tao \& Katz (1997) and Kleissl et al. (2003), one approach to check the quality of the measurements and computation algorithms is to estimate the error in reproducing the divergence-free condition $\left(\partial \widetilde{u}_{i} / \partial x_{i}=0\right)$ of the filtered-velocity field by estimating the non-dimensional parameter

$$
\eta=\frac{((\partial \widetilde{u} / \partial x)+(\partial \widetilde{v} / \partial y)+(\partial \widetilde{w} / \partial z))^{2}}{(\partial \widetilde{u} / \partial x)^{2}+(\partial \widetilde{v} / \partial y)^{2}+(\partial \widetilde{w} / \partial z)^{2}} .
$$

Ideally, this parameter should be zero since the numerator for a divergence-free velocity field is exactly zero. The upper bound on the parameter is 3 and is attained when the three velocity derivatives are equal (which obviously points to significant errors). For data that are normally distributed, with a mean of zero, the median of $\eta$ tends to 1.18 and the mean to 1.27 .

The parameter $\eta$ was computed for the derivatives obtained at the SnoHATS experiment for each data point (at $20 \mathrm{~Hz}$ ) and its probability density function (PDF) is displayed in figure 3. The median of $\eta$ was 0.29 and its mean 0.6 (significantly lower than the values for normally distributed random data). Most importantly, as can be seen in the figure, the most likely value of $\eta$ was zero and the bulk of the data points had low values; $61 \%$ of the data had $\eta<0.5$. These results are very similar to the results obtained in Kleissl et al. (2003) and can be considered satisfactory for the analysis performed in this paper. High values of $\eta$ could be due to measurement errors or to errors associated with the finite-differences computations of the gradients. As noted by Kleissl et al. (2003) for a relatively similar set-up, finite-differences errors are probably highest for the vertical-gradient component since it is computed using first order, one-sided finite differences. 


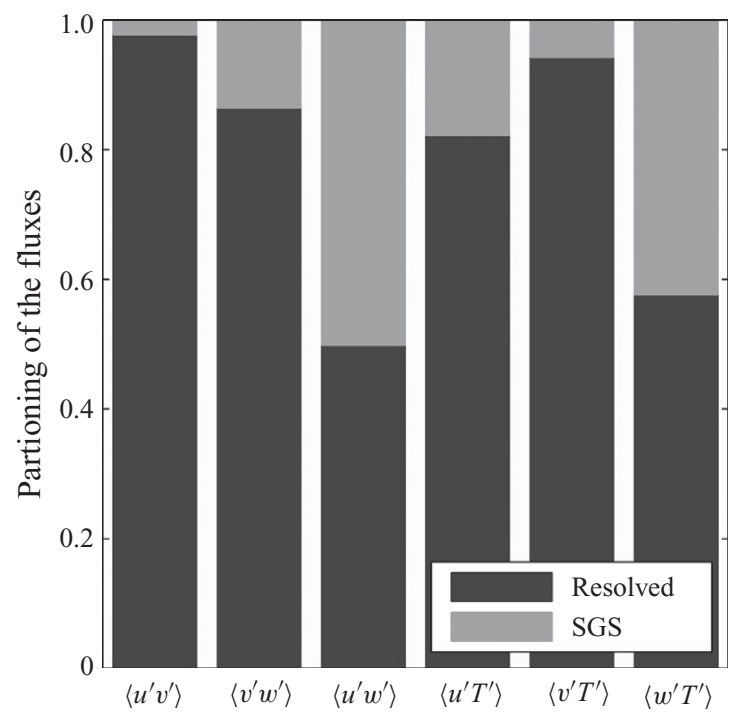

FIGURE 4. Fractions of fluxes carried by the resolved and SGSs, normalized by the total flux.

\section{Fractions of the fluxes carried by the subgrid scales}

As mentioned earlier, the main premise of LES is that the fluxes of energy, momentum and scalars are predominantly carried by the large scales. This is correct if the filter scale falls in the inertial subrange everywhere in the modelling domain; under such conditions the averages of all SGS fluxes (but not their instantaneous values) are small and approach zero asymptotically as the filter scale decreases further. Under such conditions, and as is often pointed out (e.g. Lilly 1967; Meneveau \& Katz 2000), the most important function of the SGSs is to dissipate/extract TKE or scalar variance from the resolved scales, a point we will elaborate later on.

In most simulations, however, the filter or grid-scale falls outside of the inertial range, at least in some parts of the domain. When the filter scale is in the production range, the SGSs can contribute a significant fraction of the total fluxes; one example is the near-wall region in wall-bounded flows with no grid refinement to resolve the viscous sublayer or the near-wall geometry. We are interested in computing these fractions under stable conditions and, most importantly, in assessing whether the SGS fractions increase with increasing stability. To that end, we can compute the Reynolds stresses or heat fluxes (for example $\left\langle u^{\prime} w^{\prime}\right\rangle=\langle u w\rangle-\langle u\rangle\langle w\rangle$ ) and the averages of the SGS stresses or heat fluxes as defined in (2.1) and (2.3) from the filtered velocities (e.g. $\left\langle\tau_{13}\right\rangle=\langle\widetilde{u w}\rangle-\langle\widetilde{u} \widetilde{w}\rangle$ ). Then, we compute the resolved fluxes as the difference between the Reynolds and average SGS fluxes.

In figure 4, the resolved and SGS fractions of the momentum and heat fluxes, averaged over all data from SnoHATS are compared. As can be seen, the contribution of the SGSs is lowest for the cross-stream fluxes and highest for the vertical fluxes. For vertical fluxes, the SGS contribution is very significant: $50 \%$ for the momentum flux and $42 \%$ for the vertical heat flux. This underlines one of the main difficulties in LES of very high Reynolds number flows, as compared to LES at moderate Reynolds numbers, where the viscous sublayer can be resolved: the reduction in the integral scale of turbulence close to the wall down to scales that are comparable to the grid scale effectively pushes the simulation towards a RANS simulation (in RANS, $100 \%$ 
of the stresses would be parameterized/SGS). These results reconfirm the importance of wall models (Piomelli \& Balaras 2002; Chamorro \& Porte-Agel 2009; Brasseur \& Wei 2010) in LES of high-Reynolds-number wall-bounded flows, and the need for SGS models that can adjust to the variation of the integral scale, relative to the grid scale, as the wall is approached. Another important observation in figure 4 is that the vertical fluxes seem to be the least well resolved, followed by the streamwise fluxes. This suggests that the flux-carrying eddies are anisotropic with length scales $L_{y}>L_{x}>L_{z}$; a more thorough analysis (computing the integral scales $L_{y}, L_{x}, L_{z}$ which is not possible with our data set) is needed to fully elucidate these variations between the components.

Another important parameter that controls the fraction of the fluxes falling in the SGS range is the filter scale $\Delta$. For $\Delta<z / 2$ (where $z$ is the height above the ground), the filter scale is expected to be in the inertial range (e.g. Katul, Hsieh \& Sigmon $1997)$ and the average SGS fluxes are expected to tend to zero. When $\Delta$ approaches and then increases beyond $z$, the filter scale moves into the production range and the SGS fluxes become important. The basic filter scale of SnoHATS used everywhere in this paper is $\Delta=D \cos (\alpha)$, where $D=4 \times \mathrm{d} y=3.2 \mathrm{~m}$ is the cross-stream scale of the sonic groups in figure 2 and $\alpha$ is the angle of the horizontal wind and the streamwise sonic axis (see Porte-Agel et al. 2000b). Thus, SnoHATS has a basic filter scale greater than the height above the ground (which ranged from 0.68 to $2.62 \mathrm{~m}$ ); this was intended since we are more interested in investigating the (challenging) limit, where the filter scale is in the production range which occurs when $\Delta>z$ and which is very relevant in the atmospheric surface layer.

The filter scale was also varied to test its effect on the SGS fractions of the fluxes and the TKE; this was done by changing the streamwise filter scale $\Delta_{s w}$, since the cross-stream scale is fixed by the set-up as $\Delta_{c s}=D \cos (\alpha)$; the effective filter scale was then computed as $\Delta=\left(\Delta_{s w} \Delta_{c s}\right)^{1 / 2}$. The effect of the filter scale on the SGS fractions is depicted in figure 5. As expected, the SGS fractions increase with increasing $\Delta / z$. An interesting aspect of the results is that the increase is much faster for the SGS vertical heat flux than for the SGS vertical momentum flux, essentially suggesting that scales in between the smallest and largest filter scale are very active in the vertical transport of heat, but less active in the transport of momentum. Also one can note that the SGS fractions for the vertical fluxes are much higher than for the TKE; this is consistent with the $-5 / 3$ decay rate of the spectra and the $-7 / 3$ decay rate of the co-spectra and with previous results suggesting that a range of energetic small eddies could have no role at all in the transport of momentum and heat. Kunkel \& Marusic (2006), for example show that the normalized co-spectra of the streamwise and vertical velocity components decays to $10 \%$ of its peak almost one decade earlier than the normalized spectra of the vertical velocity component.

The magnitudes of the total, resolved and SGS components of the different fluxes and of the TKE all show a net decreasing trend when plotted versus stability parameters such as $z / L_{M O}$ or $z / L_{O Z}$, where $z$ is the measurement height, $L_{M O}$ is the Obukhov length scale and $L_{O Z}$ is the Ozmidov length scale given, respectively, by

$$
L_{M O}=\frac{-u_{*}^{3}}{\kappa\left(g / \bar{\theta}_{v}\right) \overline{w^{\prime} \theta_{v}^{\prime}}} \quad \text { and } \quad L_{O Z}=\left(\frac{\varepsilon}{N_{B V}^{3}}\right)^{1 / 2}
$$

In the standard definitions above, $u_{*}$ is the friction velocity, $g=9.81 \mathrm{~m} \mathrm{~s}^{-2}$ is the gravitational acceleration, $\kappa=0.4$ is the von Kármán constant, $\varepsilon$ is the dissipation 


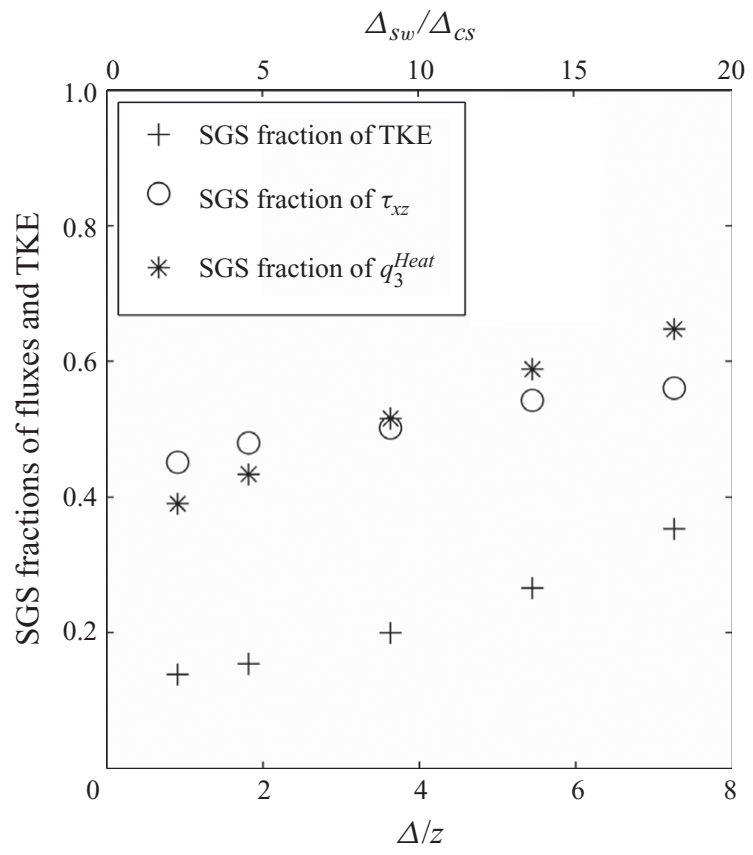

FIGURE 5. SGS fractions of the TKE and of the vertical fluxes of heat $\left(q_{3}^{\text {Heat }}\right)$ and momentum $\tau_{x z}$ as a function of filter size (lower $x$-axis) and filter aspect ratio (upper $x$-axis).

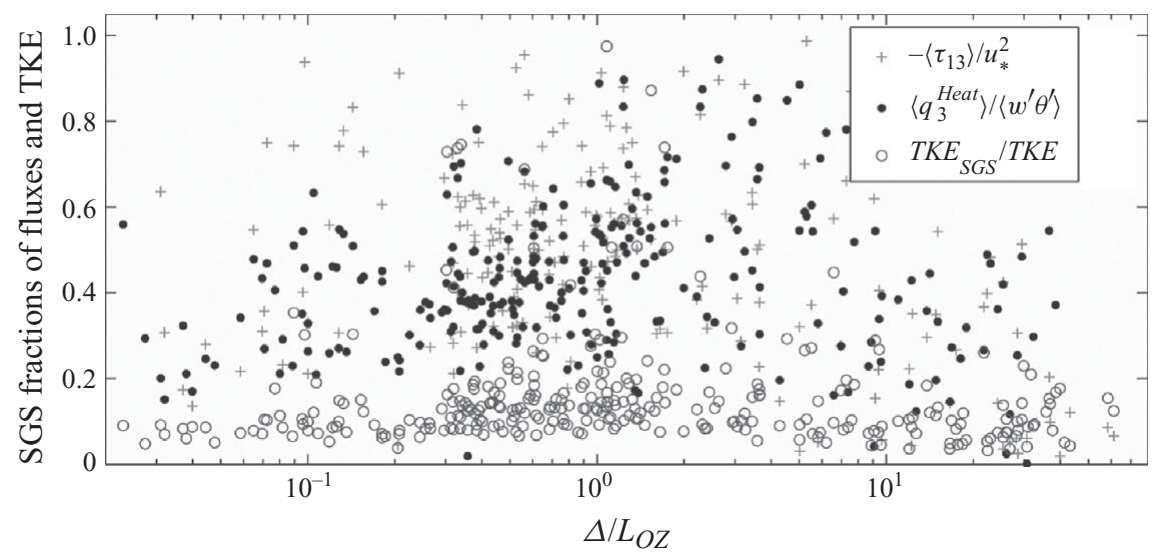

FIGURE 6. Fractions of fluxes and TKE carried by the resolved and SGS scales, normalized by the total flux.

rate (the method of computation and results for $\varepsilon$ are presented in $\S 7$ ) and $N_{B V}=$ $\left[\left(g / \bar{\theta}_{v}\right)\left(\mathrm{d} \bar{\theta}_{v} / \mathrm{d} z\right)\right]^{1 / 2}$ is the Brunt-Väisälä frequency.

However, if the role of the SGSs becomes more important with increasing stability, the fraction of the SGS fluxes, as a per cent of the total fluxes, should increase. These fractions are plotted in figure 6 for the TKE and the vertical momentum and heat fluxes, versus the stability parameter $\Delta / L_{O Z}$. This stability parameter is especially relevant in our analysis since in a stable boundary layer the Ozmidov scale is the limit between the buoyancy subrange and the inertial range; i.e. it is the smallest scale 
of turbulence that will be affected by buoyancy (Dalaudier \& Sidi 1990). Thus, for $\triangle / L_{O Z}<1$ only the resolved scales are being damped by buoyancy and as $\Delta / L_{O Z}$ increases beyond 1, the effect of buoyant forces reaches the SGSs. As can be seen, no trend can be detected. We tested these results for other flux components and stability measures and also found no clear trends as the stability increases. We then tested the sensitivity of these findings to various computational details (filter size, data correction, filter type, etc.) and consistently found no trend in the plots. The only very weak trend that can be detected is a peak in the SGS fractions around $\Delta / L_{O Z}=1$, where the effect on the buoyancy is affecting all the resolved scales but none of the SGS scales. Nevertheless, the scatter clearly indicates that buoyancy is far from being the dominant mechanism in the distribution of fluxes and TKE between the resolved and subgrid ranges.

Here our findings appear at first to contradict previous findings by Sullivan et al. (2003); however, a closer inspection of their figures and analysis reveals that the significant effect of stability on SGS flux fractions they detected is largely limited to unstable conditions. In Sullivan et al. (2003), stability is represented using the scale where the energy spectra peaks, normalized by the filter size, and hence both stable and unstable data points are lumped in their figures related to the SGS fractions. Their discussion reveals that stable conditions mostly occur when their stability parameter is less than 1; in that limit their SGS fractions depict no clear trends with increasing stability. They do, however, clearly illustrate that SGS fractions strongly decrease under unstable condition as the buoyant production of TKE increases. Other authors have also studied the impacts of stability on the resolved fraction of heat fluxes; Porte-Agel et al. (2001a) selected six 30 min periods to analyse the SGS and resolved fluxes. They report a very mild increase of the SGS fraction of the vertical heat flux under stable conditions, but a marked increase (from $16 \%$ to about $40 \%$ ) when the stable periods are compared to one unstable period. Their results for the streamwise SGS fractions show no clear trend with stability. As such, the evidence from this and the two other studies discussed do not point to a significant role of the stability, under neutral to statically stable conditions, in partitioning the fluxes into SGS and resolved scales. This observation will be analysed and explained further at the end of $\S 7$. However, unstable conditions, producing large organized convective structures, can significantly increase the integral scale compared to neutral and stable conditions and seem to clearly reduce the relative importance of SGSs.

\section{Subgrid-scale dissipations}

The rate of TKE dissipation by the SGSs $\langle\Pi\rangle=-\left\langle\tau_{i j} \widetilde{S}_{i j}\right\rangle$ (or scalar variance dissipation $\langle\chi\rangle=-\left\langle q_{i} \partial \tilde{\theta} / \partial x_{i}\right\rangle$ ) appears in the equation for resolved TKE (or scalar variance) as a net sink, since $\langle\Pi\rangle$ is typically positive in three-dimensional turbulence, although instantaneous energy backscatter, $\Pi<0$, is not uncommon. In neutral and statically unstable flows, $\Pi$ is the only sink of TKE. The results of LES are quite sensitive to the dissipation rates provided by SGS models (e.g. Porte-Agel et al. 2000a, b; Bou-Zeid et al. 2005).

As previously noted, an important function of the SGSs is to drain energy from the resolved scales, to model the cascade process that is observed in three-dimensional turbulence. In flows or regions of the flow where the mean SGS fluxes vanish, TKE dissipation clearly becomes the most critical function of the SGS model. Assuming Kolmogorov scaling in the fluctuations, the rate of dissipation of TKE can be 
computed from the third-order structure function $D_{u, u, u}$ :

$$
\varepsilon_{3}^{u}=-(5 / 4) r^{-1} D_{u, u, u}(r),
$$

evaluated at some appropriate value (in the inertial subrange) of $r$, the distance used to compute the structure function. The 5/4 factor is an exact and direct result of the Kolmogorov equation (Pope 2000) in locally isotropic turbulence. Alternatively, the dissipation can also be estimated from the second-order structure function $D_{u, u}$ :

$$
\varepsilon_{2}^{u}=0.3634 r^{-1}\left(D_{u, u}(r)\right)^{3 / 2} \text {. }
$$

Here, the coefficient is empirical; however, as noted by Chamecki \& Dias (2004), the second-order structure function approach sometimes is more accurate since it is less sensitive to departure from homogeneous isotropic conditions at the scale $r$ and, in practice, since the second-order structure function can be measured more accurately (it converges faster since, unlike the third-order structure function, it has no sign cancellations, also see Champagne et al. 1977 for a discussion of other techniques for determining dissipation). Similarly, the dissipation of temperature variance can be computed from the third-order mixed structure function $D_{u, \theta, \theta}$ :

$$
\varepsilon_{3}^{\theta}=-(3 / 4) r^{-1} D_{u, \theta, \theta}(r),
$$

or estimated from the second-order structure function of temperature $D_{\theta, \theta}$ (see Kaimal \& Finnigan 1994) as

$$
\varepsilon_{2}^{\theta}=0.3125 r^{-2 / 3}\left(\varepsilon^{u}\right)^{1 / 3} D_{\theta, \theta}(r)
$$

Again, in the third-order formulation the coefficient is exact (for locally isotropic turbulence), while in the second-order formulation, it is empirical.

The dissipations of TKE and temperature variance were computed from the SnoHATS data using the four definitions given above and a translation distance $r=\min (1 \mathrm{~m}, z / 2 \mathrm{~m})$. The choice of $r=1 \mathrm{~m}$ is optimal since it is usually small enough to be in the inertial range in the ABL (this condition is actually not met as we will discuss later) and large enough not to be significantly affected by measurement noise. However, when the snow accumulates and the distance to the wall decreases below $2 \mathrm{~m}$, it is not accurate to expect that the $1 \mathrm{~m}$ distance would fall in the inertial subrange; in that case we use $z / 2$ as the translation distance, where $z$ is the measurement height (point P2 in figure 2); the choice of $z / 2$ is motivated by the findings of Katul et al. (1997) under neutral stability conditions. The structure functions dissipation estimates are compared to the SGS dissipations $\langle\Pi\rangle=-\left\langle\tau_{i j} \widetilde{S}_{i j}\right\rangle$ and $\langle\chi\rangle=-\left\langle q_{i} \partial \tilde{\theta} / \partial x_{i}\right\rangle$ in figures 7 and 8 , respectively. When $z$ drops below $1.5 \mathrm{~m}$, the computations are more sensitive to measurement noise; we have verified that most of the scatter in these figures is from measurements with $z<1.5 \mathrm{~m}$, but we still present all the data in the figures. Note that the temperature-variance dissipation seems to be significantly more sensitive to the measurement height than the TKE dissipation.

Furthermore, to test the sensitivity of the result to our ad hoc choice of $r$, we sought to present a confidence interval for the results. To that end, we computed the dissipation based on the second- and third-order structure functions using a varying $r$ that ranges from $(0.05 s \times\langle u\rangle)$ to $(2 s \times\langle u\rangle)$. Our previous choice $r=\min (1 \mathrm{~m}, z / 2 \mathrm{~m})$, as well as the filter scale, $\Delta$, invariably fall in this range. Based on these computations (40 samples with different $r$ values for each analysis periods), we determined the $99 \%$ confidence interval for the dissipation estimates we computed for each analysis period. To display the results clearly, we fitted a linear curve through these upper and lower confidence interval bounds for each period, and plotted these lines in 


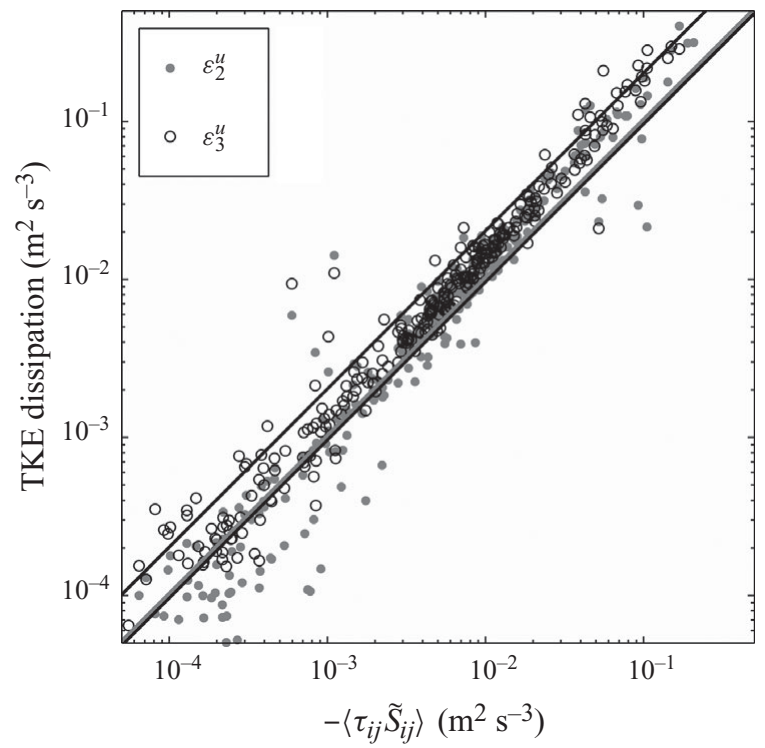

FIGURE 7. Estimates of viscous dissipation of TKE versus SGS dissipation. The black lines are the $99 \%$ confidence interval limits for the second-order structure function dissipation and the grey line (not very visible here) is the one-to-one line.

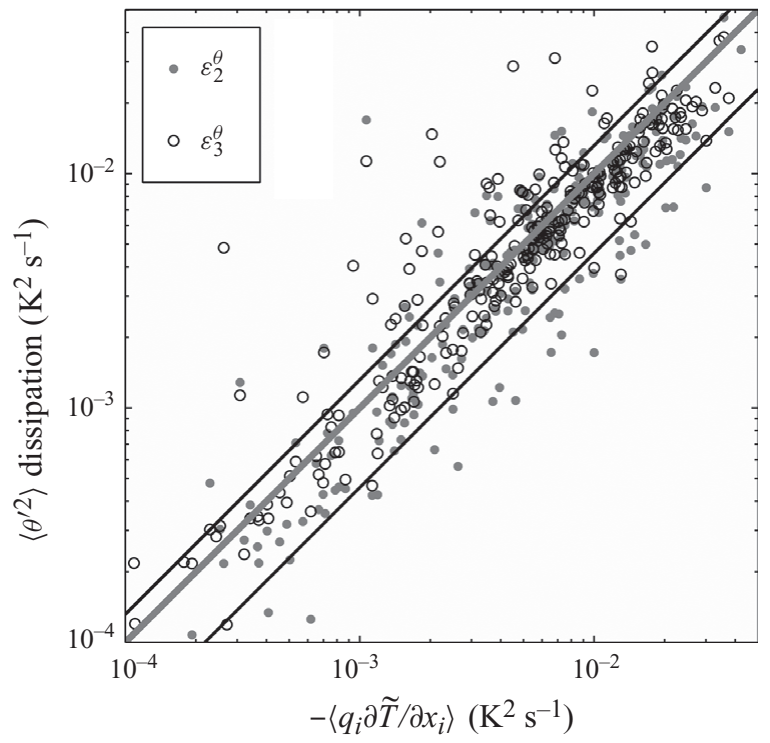

FIGURE 8. Estimates of viscous dissipation of temperature variance versus SGS dissipation. The black lines are the $99 \%$ confidence interval limits for the second-order structure function dissipation and the grey line is the one-to-one line.

figures 7 and 8, only for the second-order structure function to avoid cluttering the plot. The same analysis was done for the third-order structure function dissipation estimate and the range of confidence was similar. The points located outside of these intervals are, with $99 \%$ confidence, outliers; however, most of the points lie inside 
the confidence interval. Also note that since the filter scale $\Delta$ lies inside the range of $r$ values that we tested, a choice of $r=\Delta$ would still have yielded dissipation estimates that agree relatively well with the SGS cascades, despite the fact that $\Delta$ clearly lies in the production range. We have confirmed this by computing the dissipations with $r=\Delta$ (not shown here) and comparing them to the SGS cascades of TKE and scalar variance.

The good agreement between the SGS TKE cascade and the estimates of the viscous dissipation using the structure functions is in fact surprising at first; especially as no attempt was made to filter out periods where $r$ cannot be assumed to be in the inertial range and that buoyant destruction is expected to play an important role at both resolved and SGS scales. When the dissipation estimates were plotted versus the variable $r$ used to compute the confidence intervals, the dissipations for a large number of periods varied with $r$ and did not display any regions of constant values, which would have been expected if inertial range scaling was applicable. A more in depth analysis does, however, explain this good agreement in figures 7 and 8 .

In a stable surface layer under steady state and horizontally-homogeneous conditions like the ones studied here, turbulent and pressure transports of TKE are generally found to be negligible (though this assumption does not influence our analysis); this reduces the mean TKE conservation equation to a balance between the means of mechanical production, $\langle P\rangle$, as a source and buoyant destruction, $\langle B\rangle=\left(-g / \overline{\theta_{v}}\right)\left(\left\langle w \theta_{v}\right\rangle-\langle w\rangle\left\langle\theta_{v}\right\rangle\right)$, and viscous dissipation, $\langle\varepsilon\rangle$, as sinks (Wyngaard 1992) following:

$$
\langle P\rangle=\langle\xi\rangle=\langle\varepsilon\rangle+\langle B\rangle,
$$

where $\langle\xi\rangle$ is the total mean TKE dissipation/destruction by viscous and buoyant effects. For the SGS part of the TKE, $k_{S G S}=\left(\widetilde{u_{i} u_{i}}-\widetilde{u}_{i} \widetilde{u}_{i}\right) / 2$, the evolution equation is given by (Lilly 1967; Moeng 1984; Sagaut 2006)

$$
\frac{\partial k_{S G S}}{\partial t}+\widetilde{u}_{j} \frac{\partial k_{S G S}}{\partial x_{j}}=-\frac{1}{2} \frac{\partial Q_{j}}{\partial x_{j}}-\varepsilon-\tau_{i j} \widetilde{S}_{i j}+\frac{g}{\overline{\theta_{v}}}\left(\widetilde{w \theta_{v}}-\widetilde{w} \widetilde{\theta_{v}}\right),
$$

where $Q_{j}$ represents spatial fluxes from SGS motions, pressure and viscous effects, and $\varepsilon$ is the instantaneous viscous dissipation of TKE. Upon Reynolds averaging the above equation, for steady state, horizontally-homogeneous flows, and since for filter scales near the inertial range the vertical gradients are expected to be small, the left-hand side of (7.6) reduces to zero and the horizontal gradients of the spatial fluxes also vanish. Therefore, the conservation of SGS TKE reduces to a balance between the energy flux across the filter scale $\langle\Pi\rangle=-\left\langle\tau_{i j} \widetilde{S}_{i j}\right\rangle$ as a source, and the SGS buoyant destruction (the mean of the last term times $\left.-1,\left\langle B_{S G S}\right\rangle=-\left(g / \overline{\theta_{v}}\right)\left\langle\widetilde{\left\langle\theta_{v}\right.}-\widetilde{w} \widetilde{\theta_{v}}\right\rangle\right)$ and the mean viscous dissipation $\langle\varepsilon\rangle$ as sinks (Sullivan, McWilliams \& Moeng 1994). This balance can be written as

$$
\langle\Pi\rangle=\left\langle B_{S G S}\right\rangle+\langle\varepsilon\rangle .
$$

Here, note that the SGS dissipation term $\langle\Pi\rangle$ is in reality an energy-cascade process due to the extraction of TKE from the resolved scales (or directly from the mean flow) by the SGS scales (it represents mechanical TKE production by the SGSs), while the buoyant destruction terms $B$ and $B_{S G S}$ physically represent the conversion of TKE into mean potential energy in the flow (see Randall 1984 for a more thorough discussion); the viscous dissipation represents a conversion of kinetic energy into heat.

From (7.5) and (7.7), and since the buoyant destruction can be divided into a fraction occurring at the SGSs $\left\langle B_{S G S}\right\rangle$ and a fraction occurring at the resolved scales 


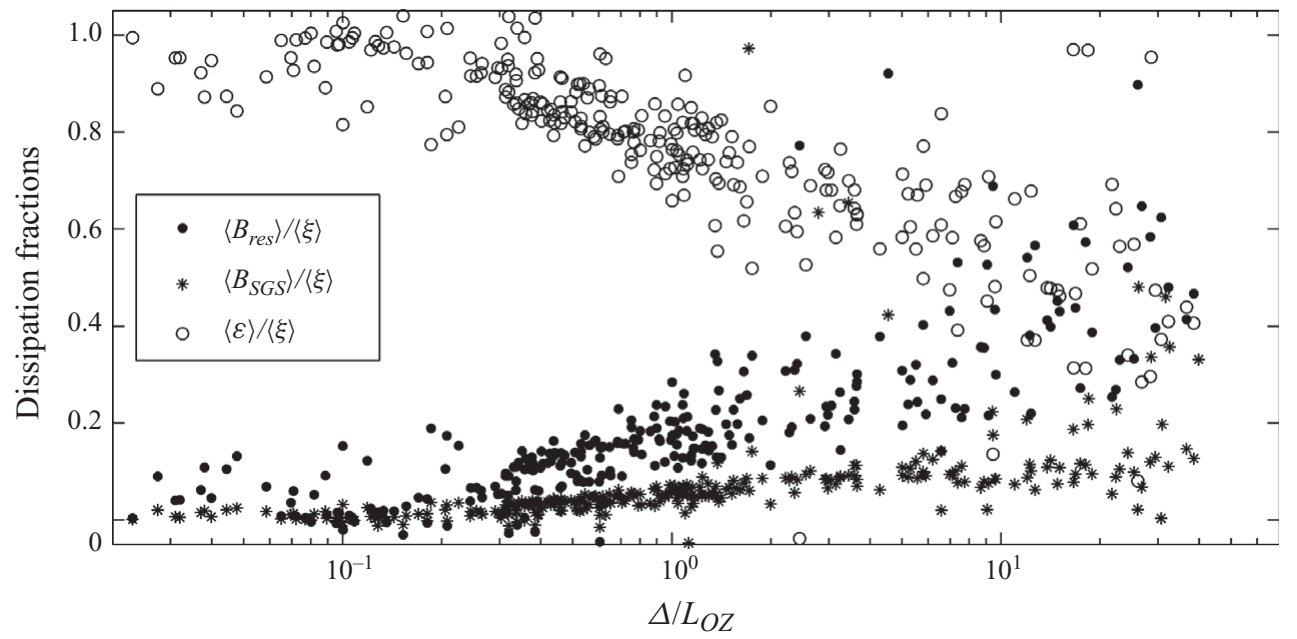

FIGURE 9. TKE dissipation/destruction by buoyancy in the resolved range, $\left\langle B_{\text {res }}\right\rangle$, and in the SGS range, $\left\langle B_{S G S}\right\rangle$, and by viscous dissipation, $\langle\varepsilon\rangle$, as a fraction of total dissipation $\langle\xi\rangle$ (some values of $\varepsilon$ slightly larger than 1 correspond to negative buoyant (production) terms and could be due either to real upward heat fluxes despite the negative gradients suggested by $\Delta / L_{O Z}$ or to measurement errors).

$\left\langle B_{\text {res }}\right\rangle$, we can rewrite $(7.5)$ as

$$
\langle P\rangle=\langle\xi\rangle=\langle\Pi\rangle+\left\langle B_{\text {res }}\right\rangle=\langle\varepsilon\rangle+\left\langle B_{S G S}\right\rangle+\left\langle B_{\text {res }}\right\rangle,
$$

which illustrates that the produced TKE is eventually removed (locally) by: (i) viscous dissipation at the SGSs, (ii) buoyant destruction at the resolved scales or (iii) buoyant destruction at the SGSs. Hence, based on (7.7), the good match in figure 7 between $\langle\Pi\rangle$ and the viscous dissipation estimates might be interpreted (erroneously as will be shown later) as implying that the SGS buoyancy destruction term is negligible. To verify this, we directly computed the buoyancy destruction of SGS-TKE term $\left\langle B_{S G S}\right\rangle$ according to its definition from the vertical velocity and temperature. We also computed the resolved buoyancy destruction term as the residual $\left\langle B_{r e s}\right\rangle=\langle B\rangle-$ $\left\langle B_{S G S}\right\rangle$. We then estimated the viscous dissipation directly from (7.7) as $\langle\varepsilon\rangle=\langle\Pi\rangle-$ $\left\langle B_{S G S}\right\rangle$.

The three dissipation/destruction terms $\left\langle B_{\text {res }}\right\rangle,\left\langle B_{S G S}\right\rangle$ and $\langle\varepsilon\rangle$ are plotted versus the stability parameter $\Delta / L_{O Z}$ in figure 9 . As could be noted, at low stabilities, viscous dissipation is about two orders of magnitude larger than the two buoyant destruction terms; however, the importance of buoyant destruction significantly increases at higher stabilities. At very high stabilities, the three terms are on the same order, but the SGS destruction remains lower than the other two terms. The results further indicate that the SGS-TKE cascade term $\left(\langle\Pi\rangle=\left\langle B_{S G S}\right\rangle+\langle\varepsilon\rangle\right)$ remains the main sink of TKE from the resolved scales since in our data it was always at least $50 \%$ larger than the resolved buoyancy destruction. Therefore, the main goal of an SGS model still has to be the correct prediction of the TKE cascade term $\Pi$. At high stabilities, this cascading energy is eventually destroyed through both viscous dissipation and SGS buoyant destruction.

When figure 7 is interpreted in the light of these results, one must conclude that the good agreement in that figure is due to the fact that the estimates of the viscous dissipations from the structure functions are in reality good measures of the energy 
cascade across the scale of the translation distance $r$. This fact becomes obvious when the derivation of the structure function estimates of the dissipation is revisited. In the reduction of the Kármán-Howarth equation to the Kolmogorov equation that yields the third-order structure function-dissipation relation, the transient and viscous diffusion terms are neglected leaving the inertial (cascade) and dissipation terms (Pope 2000). Hence this equation fundamentally implies that the cascade (at scale $r$ ) is equal to the viscous dissipation and the latter can be estimated by the former in the inertial subrange, where Kolmogorov's assumption of homogeneous isotropic turbulence holds. In stable flows and when using a translation distance $r$ that might lie in a range affected by buoyancy or turbulence production, these estimates continue to be good estimates of the energy cascade at that scale $r$.

In contrast to the dissipation of TKE, the dissipation of temperature variance is not affected by buoyancy and the mean cascade of temperature variance from the resolved scales, which again is the SGS temperature variance production (estimated as $\varepsilon_{2}^{\theta}, \varepsilon_{3}^{\theta}$, or $\langle\chi\rangle=-\left\langle q_{i} \partial \widetilde{\theta} / \partial x_{i}\right\rangle$ as in figure 8) should match the viscous dissipation. This is confirmed by the better agreement of temperature variance cascade and dissipation in figure 8 (despite the higher scatter, the data fall on the 1-to-1 line) compared to the TKE cascade and dissipation in figure 7.

Figure 9 also prompts us to revisit the findings on the SGS flux and variance dependence on stability. It is clear from figure 9 that for $\Delta / L_{O Z}<1$ (where only the resolved scales are affected by buoyancy), total buoyant destruction amounts to less than $20 \%$ of the total TKE dissipation and the effect of buoyancy on the flow is very moderate. As the effect of buoyancy increases, $L_{O Z}$ decreases below $\Delta$ and the buoyancy now impacts the resolved as well as the SGS scales. Hence our findings regarding the SGS fractions' lack of sensitivity to stability are not surprising; when the stability is strong enough to be significant, it is damping both the resolved as well as the largest, most important, SGSs.

Furthermore, the classic results for the variation of the spectra and co-spectra in the stable ABL (Kaimal et al. 1972) indicate that, as the stability increases: (i) all fluxes and variances carried by production range eddies are damped, (ii) the normalized inertial range co-spectra remain unchanged but the frequency of transition from the buoyancy to the inertial range increases, corresponding to a reduction in the Ozmidov scale and (iii) the spectral slopes (before and after the peak) largely remain constant suggesting that scales larger than about $5 L_{O Z}$ are damped similarly (the proportional drops in their spectra are equal). When the results presented here are analysed in that context, we are able to reach three conclusions. First, with a filter size of about $3.2 \mathrm{~m}$ or about twice the distance to the ground surface, which is representative of current resolutions in LES of ABL flows, the largest flux-carrying eddies are still resolved. Even under the strongest stabilities encountered during SnoHATS, these resolved scales account for at least $50 \%$ of the total vertical fluxes (more for horizontal fluxes). Under even higher stabilities, or for large filter sizes, this result might not hold. Second, we observed a reduction in both the resolved and SGS stresses and fluxes as the stability increases, confirming the analysis based on the Ozmidov scale and the TKE dissipation fractions that when the static stability is significant, it affects turbulence well into the unresolved scales. While the resolved eddies might be damped more significantly than SGS eddies when $0.1 \Delta<L_{O Z}<\Delta$, the difference in the damping is not large enough to shift the fluxes significantly to smaller scales or higher frequencies. Finally, stability is not the most critical factor in determining the partitioning of the fluxes into resolved and SGS parts as illustrated by the scatter in the results in figure 6 . The filter size is more relevant for the relative subgrid/resolved partitioning. 


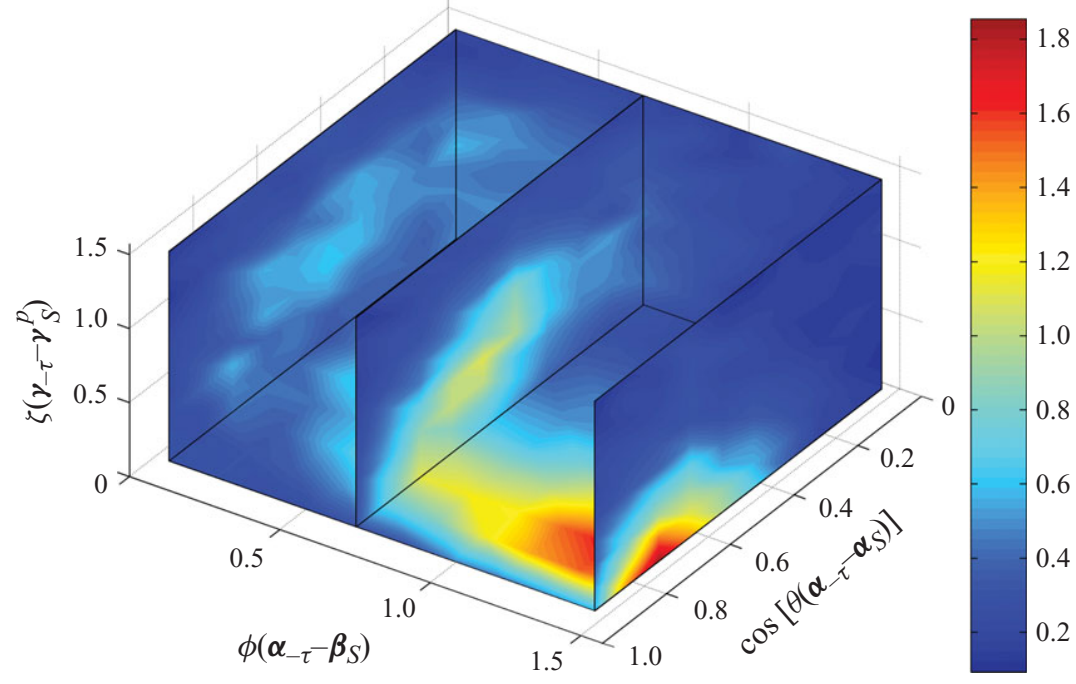

FIGURE 10. Relative alignment of the eigenvectors of the SGS stress tensor and the strain-rate tensor.

\section{Stress-strain-rate alignment}

To further investigate relationships between filtered and resolved quantities, the alignment between the SGS stress tensor and the filtered strain-rate tensor is considered. To determine the three-dimensional relative orientation of these two symmetric and traceless tensor eigensystems, we must specify three unique angles, as explained in detail in Tao, Katz \& Meneveau (2002) and Higgins, Parlange \& Meneveau (2003). The extensive eigendirection of the (negative of the) SGS stress, $\boldsymbol{\alpha}_{-\tau}$ is expressed in spherical coordinates relative to the strain-rate eigendirections $\left(\boldsymbol{\alpha}_{\tilde{S}}, \boldsymbol{\beta}_{\widetilde{S}}\right.$ and $\left.\boldsymbol{\gamma}_{\widetilde{S}}\right)$, thus specifying two of the three unique angles

$$
\theta=\cos ^{-1} \frac{\left|\boldsymbol{\alpha}_{\tilde{S}} \cdot \boldsymbol{\alpha}_{-\tau}\right|}{\left|\boldsymbol{\alpha}_{\widetilde{S}}\right|\left|\boldsymbol{\alpha}_{-\tau}\right|} \quad \text { and } \phi=\cos ^{-1} \frac{\left|\boldsymbol{\beta}_{\widetilde{S}} \cdot\left(\boldsymbol{\alpha}_{-\tau}-\left(\boldsymbol{\alpha}_{\widetilde{S}} \cdot \boldsymbol{\alpha}_{-\tau}\right) \boldsymbol{\alpha}_{\widetilde{S}}\right)\right|}{\left|\boldsymbol{\beta}_{\widetilde{S}}\right|\left|\left(\boldsymbol{\alpha}_{-\tau}-\left(\boldsymbol{\alpha}_{\widetilde{S}} \cdot \boldsymbol{\alpha}_{-\tau}\right) \boldsymbol{\alpha}_{\widetilde{S}}\right)\right|},
$$

but the orientation of the SGS stress relative to the filtered strain rate has not been fixed completely since the compressive, $\boldsymbol{\gamma}_{-\tau}$, and intermediate, $\boldsymbol{\beta}_{-\tau}$, eigendirections are free to rotate in a plane perpendicular to $\boldsymbol{\alpha}_{-\tau}$. The compressive eigendirection of the filtered strain rate, $\boldsymbol{\gamma}_{\tilde{S}}$, is projected into the plane perpendicular to the fixed SGS stress eigendirection, $\boldsymbol{\alpha}_{-\tau}$. This projection now lies in the plane spanned by two eigendirections of the SGS stress $\boldsymbol{\gamma}_{-\tau}$ and $\boldsymbol{\beta}_{-\tau}$, and its angle relative to the compressive direction of the SGS stress $\boldsymbol{\gamma}_{-\tau}$

$$
\zeta=\cos ^{-1} \frac{\left|\boldsymbol{\gamma}_{-\tau} \cdot\left(\boldsymbol{\gamma}_{\tilde{S}}-\left(\boldsymbol{\gamma}_{\tilde{S}} \cdot \boldsymbol{\alpha}_{-\tau}\right) \boldsymbol{\alpha}_{-\tau}\right)\right|}{\left|\boldsymbol{\gamma}_{-\tau}\right|\left|\left(\boldsymbol{\gamma}_{\tilde{S}}-\left(\boldsymbol{\gamma}_{\widetilde{S}} \cdot \boldsymbol{\alpha}_{-\tau}\right) \boldsymbol{\alpha}_{-\tau}\right)\right|}
$$

will fix the relative position of the two tensors (see sketch in Higgins et al. 2003).

Figure 10 displays the three-dimensional joint PDF of the alignment angles between the filtered strain rate and SGS stress. This tensor alignment is quite similar in structure to the alignment trends seen in Higgins et al. $(2003,2007)$ and Tao et al. (2002). Structurally, this alignment shows that the eigensystems tend to be misaligned 


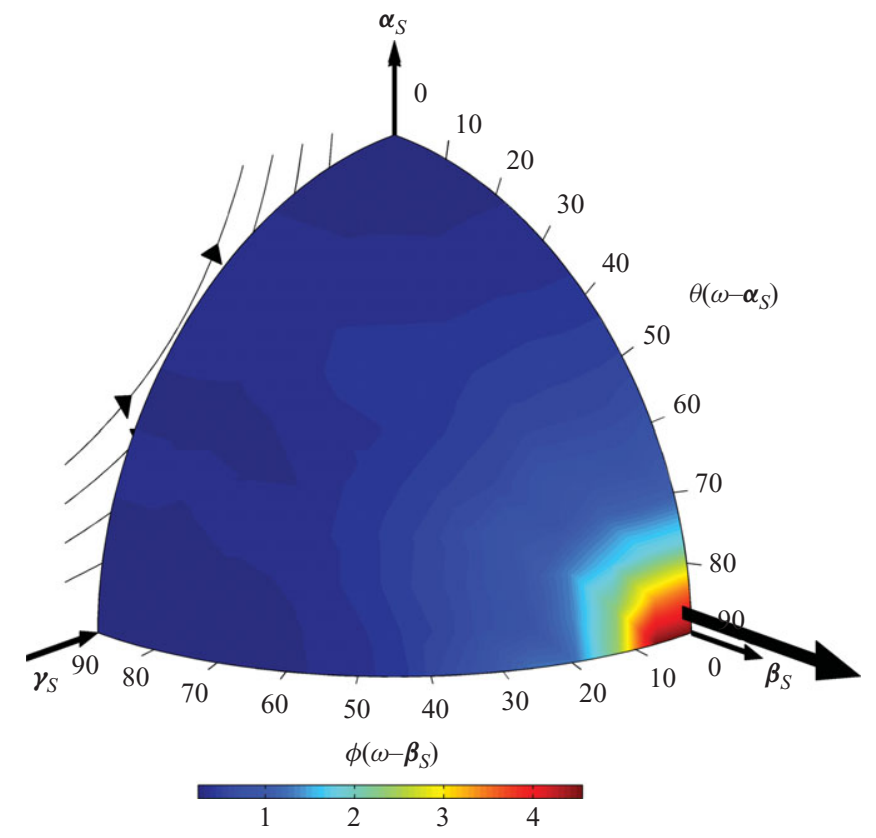

FIGURE 11. Alignment of the vorticity with the strain rate tensor eigenvectors.

by about $35^{\circ}$ off the most extensive strain-rate eigendirection (rather than to display an eddy-viscosity alignment), but also that this misalignment is structurally persistent. This persistent alignment has now been observed in atmospheric flow over agricultural fields (Higgins et al. 2003; Tao et al. 2002), over the idealized conditions of the Utah salt flats (Higgins et al. 2007) and in turbulent flow in a square duct (Tao et al. 2002). Furthermore, with this analysis, the persistent alignment trend has now been observed to occur under the full range of static stabilities. However, note that some studies found evidence that the alignment between the SGS heat flux and temperature gradients vary with stability (Chamecki 2010), suggesting that Smagorisnky-type models might not be the best option for SGS heat flux modelling.

A geometric analysis among filtered variables is presented in figure 11. Here, the filtered vorticity vector is expressed in polar coordinates relative to the filtered strain-rate eigensystem. Investigations concerning the alignment of vorticity and the eigenvectors of the filtered strain rate have already received considerable attention. Ashurst et al. (1987) observed that the vorticity was aligned with the intermediate eigendirection, $\boldsymbol{\beta}_{\widetilde{S}}$, of the strain-rate tensor in DNS data. Tsinober, Kit \& Dracos (1992) found similar results from hot-wire measurements. Tao et al. (2002) observed the same result based on their filtered holographic particle-image velocimetry measurements. Higgins et al. (2003) also reported this alignment trend for unstable atmospheric conditions. Here, we examine the vorticity alignment to determine if this turbulence feature is present under stable conditions. Figure 11 shows a very strong peak in the measured joint PDF of angles between filtered-fluctuating vorticity and filtered-fluctuating strain-rate basis. The filtered-fluctuating vorticity is clearly preferentially aligned with the intermediate direction of the filtered-fluctuating strain rate. Thus, this phenomenological feature of turbulence appears to be independent of the stability since now this alignment is reported for both unstable (Higgins et al. 2003) and stable (present study) conditions. 


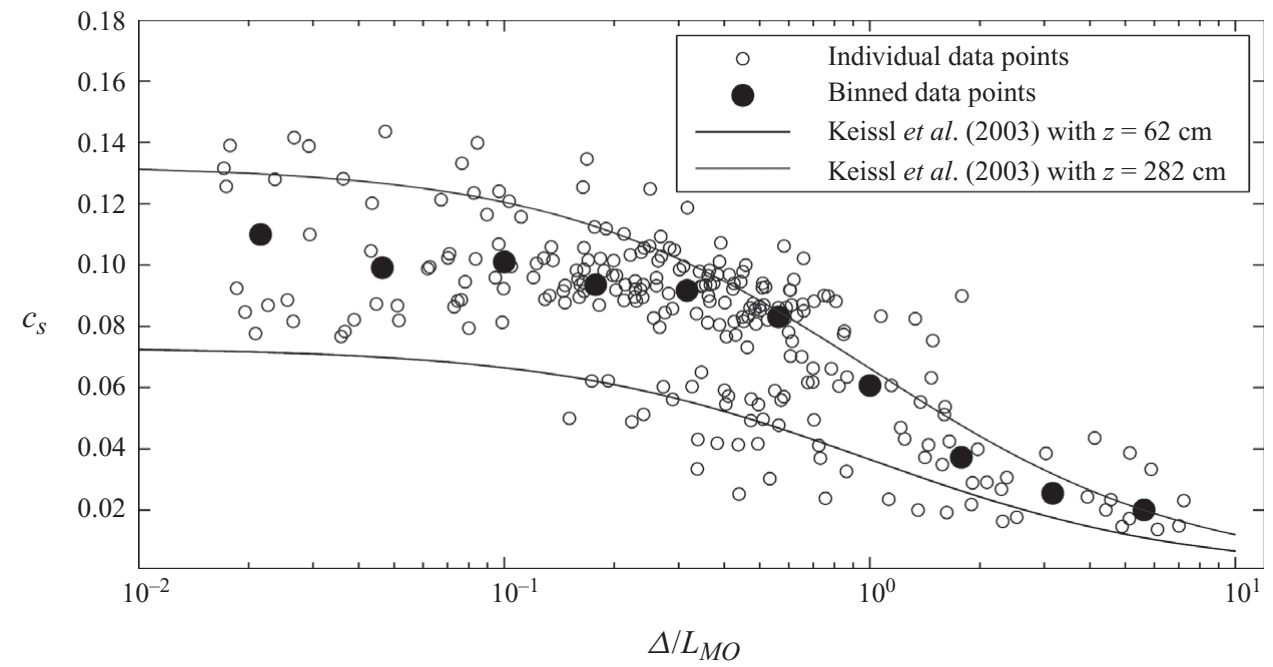

FIGURE 12. Variation of the Smagorinsky coefficient with the stability parameter based on the Obukhov scale.

\section{Model coefficients and the effect of stability}

The coefficients of the Smagorinsky model appearing in (2.4) and (2.5) are the only unknowns in the Smagorinsky model equations when performing an LES. Their values are well known for homogeneous isotropic turbulence $\left(c_{s} \approx 0.16\right.$ from Lilly 1967 and $P r_{S G S} \approx 0.47$ from Mason 1989). However, for realistic flows, different values are required and have to be known a priori for a given flow or could be determined using dynamic approaches (Germano et al. 1991; Porte-Agel et al. 2000a; Porte-Agel 2004; Bou-Zeid et al. 2005; Stoll \& Porte-Agel 2006). Determining the coefficient for a given flow, or verifying the ability of dynamic approaches to yield the correct coefficients, can be performed using an a priori model testing framework. Nevertheless, it turns out that the 'optimal' or 'correct' model coefficient is not unique and depends on what we require the model to replicate (see examples and discussion in Meneveau 1994). For many flows, the optimal model coefficients are usually determined to match the exact (measured) SGS dissipation or vertical fluxes (e.g. Kleissl et al. 2003). These choices are well justified by the analysis in this paper (figures 4, 7 and 8). More specifically, this coefficient determination is performed by matching the mean measured SGS dissipation rates of TKE $\left(\langle\Pi\rangle=-\left\langle t_{i j} \widetilde{S}_{i j}\right\rangle\right)$ or temperature variance $\left(\langle\chi\rangle=-\left\langle q_{i}^{\text {heat }} \partial \widetilde{\theta} / \partial x_{i}\right\rangle\right)$ with the modelled ones. We thus obtain the most suitable coefficients for the Smagorinsky model

$$
c_{s}^{2}=\frac{-\left\langle\tau_{i j} \widetilde{S}_{i j}\right\rangle}{2(\Delta)^{2}\left\langle\widetilde{S} \mid \widetilde{S}_{i j} \widetilde{S}_{i j}\right\rangle}, \quad \operatorname{Pr}^{-1} c_{s}^{2}=\frac{-\left\langle q_{i}^{\text {heat }}\left(\partial \widetilde{\theta} / \partial x_{i}\right)\right\rangle}{\left\langle\Delta^{2}|\widetilde{S}|\left(\partial \widetilde{\theta} / \partial x_{i}\right)\left(\partial \widetilde{\theta} / \partial x_{i}\right)\right\rangle} .
$$

These expressions can be evaluated based on the SnoHATS data. First we examine the Smagorinsky coefficient matching the kinetic energy dissipations, $c_{s}$. The coefficient is plotted in figure 12 versus the stability parameter $\Delta / L_{M O}$. Each data point corresponds to the result for a $30 \mathrm{~min}$ period. Results for the global averages for different stability bins/ranges are also shown as solid black data points. The bins were selected so that their centres are equally spaced on the logarithmic scale (the data points are plotted in the figure at the location of the bin centre). Only bins 


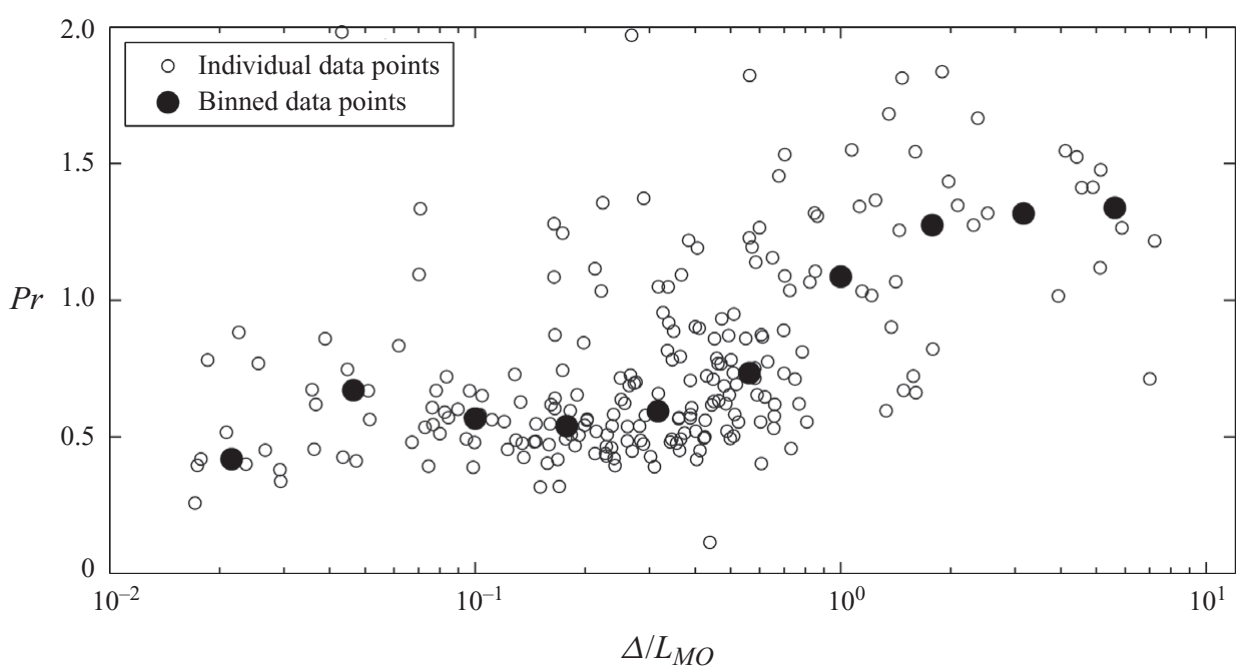

Figure 13. Variation of the SGS Prandtl number with the stability parameter based on the Obukhov scale.

with a number of individual points exceeding five were plotted since a lower number would not be sufficient to compute an average. All bins with centres in the range $10^{-2}<\Delta / L_{M O}<10^{1}$ had sufficient points.

The variation with stability is in very good agreement with the findings of Kleissl et al. (2003), who further proposed a parameterization of $c_{s}$ as a function of stability, filter scale and distance to the wall:

$$
c_{s, \text { Kleiss }}^{\Delta}=c_{o}\left[1+R\left(\frac{\Delta}{L_{M O}}\right)\right]^{-1}\left[1+\left(\frac{c_{o}}{\kappa} \frac{\Delta}{z}\right)^{n}\right]^{-1 / n},
$$

where $c_{o}=0.135$ and $n=3$ are empirical constants determined in Kleissl et al. (2003) using unconstrained optimization, $\kappa=0.4$ is the von Kármán constant and $R$ is the ramp function. This parameterization is depicted as solid lines for the minimum and maximum distances to the ground surface encountered in SnoHATS; the data from this experiment fall within the range predicted by (9.2).

In a follow-up on their work, Kleissl et al. (2004) also tested the scale-invariant dynamic model (Germano 1986) and the scale-dependent dynamic model (PorteAgel et al. 2000a; Bou-Zeid et al. 2005) for their ability to yield the correct model coefficients determined a priori through (9.1). They observed that the scale-invariant model significantly underestimated the optimal model coefficients; this is a welldocumented finding related to the fact that, near the surface, the filter scale is in the production range and the model coefficients vary with scale. On the other hand, Kleissl et al. (2004) found that the scale-dependent dynamic model gave coefficients in very good agreement with the experimental values for all stabilities. The SnoHATS set-up was not designed to perform the two test-filtering operations required for the dynamic models (even though such tests could still be performed) and hence we do not reproduce the analysis of Kleissl et al. (2004). However, the fact observed in their paper, that a scale-dependent model can reproduce experimentally determined coefficients with good accuracy under stable conditions, will be important in our discussion and conclusions later in this paper.

Figure 13 depicts the variation of the SGS Prandtl number with stability. Kleissl et al. (2003) also studied this variation but no clear trend could be observed in their 
data, probably due to a low number of data records with strong stability (few points with $\Delta / L_{M O}>3$ ). The SnoHATS data set is a useful addition in that regard since, as can be seen in figure 13, we have a significant number of data points with stabilities up to $\Delta / L_{M O} \cong 8$. The data here clearly indicate that $\operatorname{Pr}$ increases with increasing stability and reaches around 1.4 at very high stabilities. As the thermal stratification is reduced and the ABL tends to neutral, the value of $\operatorname{Pr}$ approaches 0.5 which is very close to the value of 0.47 theoretically computed by Mason (1989). Mason's value is actually the ratio of the inertial range spectral constants for temperature $\left(E_{T}=0.7 \varepsilon^{-1 / 3} \varepsilon_{T} \kappa^{-5 / 3}\right)$ and velocity $\left(E=1.5 \varepsilon^{2 / 3} \kappa^{-5 / 3}\right)$ for which we assume the same values as Mason: 0.7 and 1.5, respectively. However, note that in the neutral limit, the atmosphere is well mixed and the temperature variations are small. This will increase the relative error in the measurements of temperature variations and heat fluxes, and the computations of $\mathrm{Pr}$ in that limit are, therefore, less reliable. However, the increase in $\operatorname{Pr}$ with increasing stability is very clear and indicates that the turbulent transport efficiency of momentum is increasing relative to the transport efficiency of heat at higher stabilities. This trend is in agreement with theoretical studies of the variation of the turbulent (not only SGS) Prandtl number under stable conditions (Venayagamoorthy \& Stretch 2010) and complements the trend observed in Vercauteren et al. (2008) for the variation or $P r$ under statically unstable (convective) conditions (see Appendix); that study observed a decrease in $\mathrm{Pr}$ as the atmosphere becomes more unstable.

Despite the good agreement with previous studies, one can note significant scatter in the $30 \mathrm{~min}$ data when plotted against $\Delta / L_{M O}$. This is not entirely surprising since $L_{M O}$ is not the best scale to characterize thermal-stability effects under stable conditions as noted in previous studies (e.g. Forrer \& Rotach 1997; Handorf, Foken \& Kottmeier 1999) for several reasons. First, it is based on the ratio of fluxes $\left(u_{*}\right.$ and sensible heat flux $H$ ), which can become very small and challenging to measure at higher stabilities where the flow laminarizes (these stabilities are reached not due to a large $H$, but rather due to a vanishing $u_{*}$ ), and also at near neutral stability where $H$ is very small. Second, despite being based on $H$, the variation of $\Delta / L_{M O}$ (or $\left.z / L_{M O}\right)$ is not monotonic with $H$ since at neutral stability both are zero, at moderate stabilities they are non-zero and at very strong stabilities $H$ decreases again to very small values due to the damping of turbulence, while $\Delta / L_{M O}$ increases continuously (high values of $\Delta / L_{M O}$ are basically attained under conditions of zero wind rather than conditions of strong negative heat flux). In fact, Malhi (1995) finds that the maximum downward heat flux occurs at $z / L_{M O}$ of about 0.2 , though this value is not necessarily universal. Therefore, the turbulent flux of heat becomes an inadequate measure of stability. The effects of the non-monotonic variation of $H$ with the Monin-Obukhov stability parameter under stable conditions have also been addressed in other studies (De Bruin 1994; Basu et al. 2008). Another reason is that under stable conditions, $L_{M O}$ is a measure of mechanical turbulence production to buoyant turbulence destruction; however, as discussed before in this paper, buoyant TKE destruction seems to be a minor component in the TKE balance: under all but the most stable conditions, TKE destruction is mainly through viscous dissipation. Other effects of stability on the dynamics and structure of turbulence in the stable ABL are not accounted for in $L_{M O}$.

As such, the Ozmidov length scale $\left(L_{O Z}\right)$ might be better suited to characterize the degree of thermal stratification since it is based on the gradient of temperature which can be measured more accurately than $H$ at all stabilities and since $z / L_{O Z}$ varies monotonically with this gradient. In addition, the Ozmidov length scale is a better predictor of the largest turbulent scales in a stable flow since it is the 


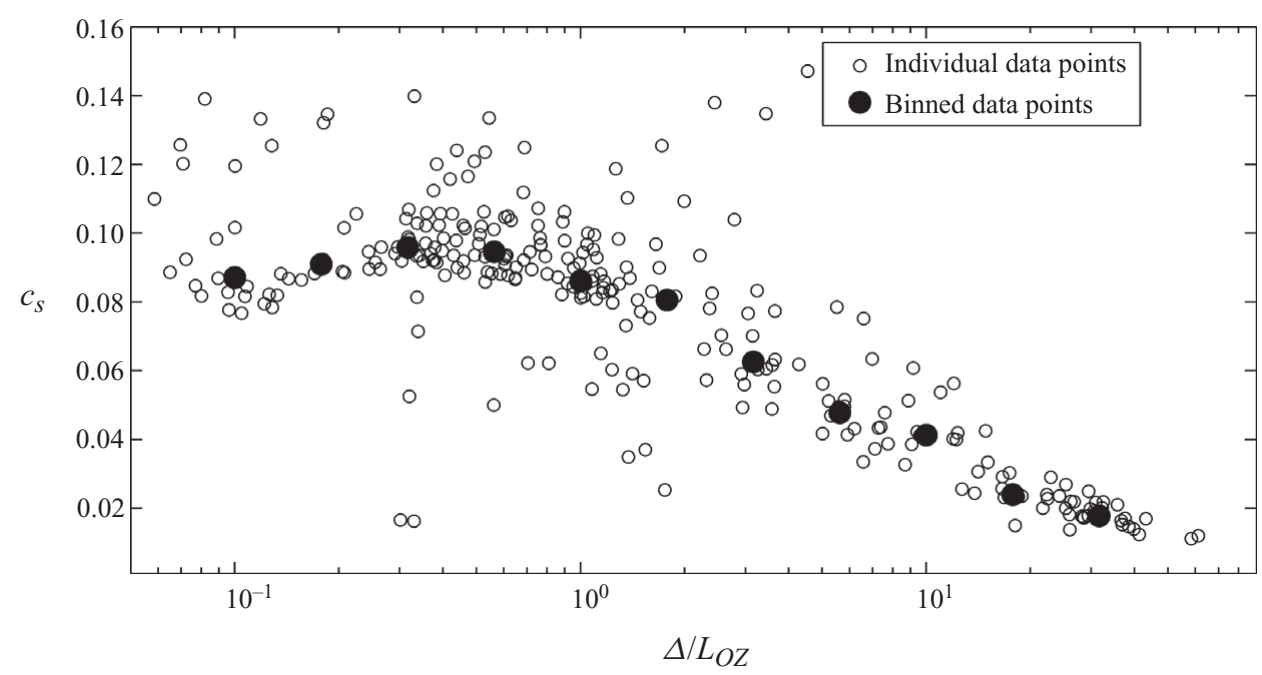

FIGURE 14. Variation of the Smagorinsky coefficient with the stability parameter based on the Ozmidov scale.

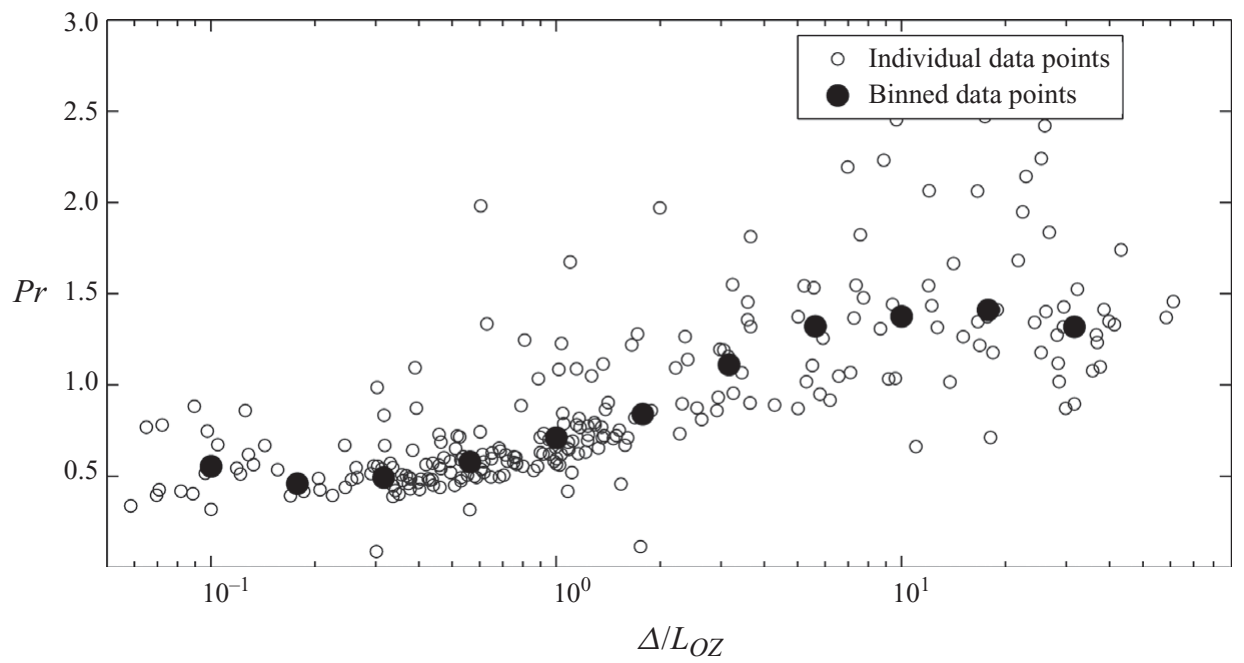

FIGURE 15. Variation of the SGS Prandtl number with stability parameter based on the Ozmidov scale.

lower limit of the buoyancy subrange that separates it from the inertial subrange (Dalaudier \& Sidi 1990). As such, $\Delta / L_{O Z}$ indicates whether the SGSs are affected by the stratification $\left(\Delta<L_{O Z}\right)$ or not $\left(\Delta>L_{O Z}\right)$. We plot the model coefficients, $c_{s}$ and $\operatorname{Pr}$, versus $\Delta / L_{O Z}$ in figures 14 and 15 . As can be noticed, there is less scatter than with $\Delta / L_{M O}$ and the trends of the coefficient are much clearer. We have also tested several other stability indices including the flux and gradient Richardson numbers, as well as scales based on the SGS TKE such as the one proposed by Deardorff (1980): $L_{k, S G S}=0.76\left(k_{S G S} / N_{B V}\right)^{1 / 2}$; we invariably found that the Ozmidov scale yielded a better collapse (lower scatter) of the data; though other gradient-based indices, such as the gradient Richardson number and $L_{k, S G S}$, also perform reasonably well especially in comparison to $z / L_{M O}$ or other flux-based parameters. The skill of the index based on the Ozmidov scale could be linked to the inclusion of the dissipation 


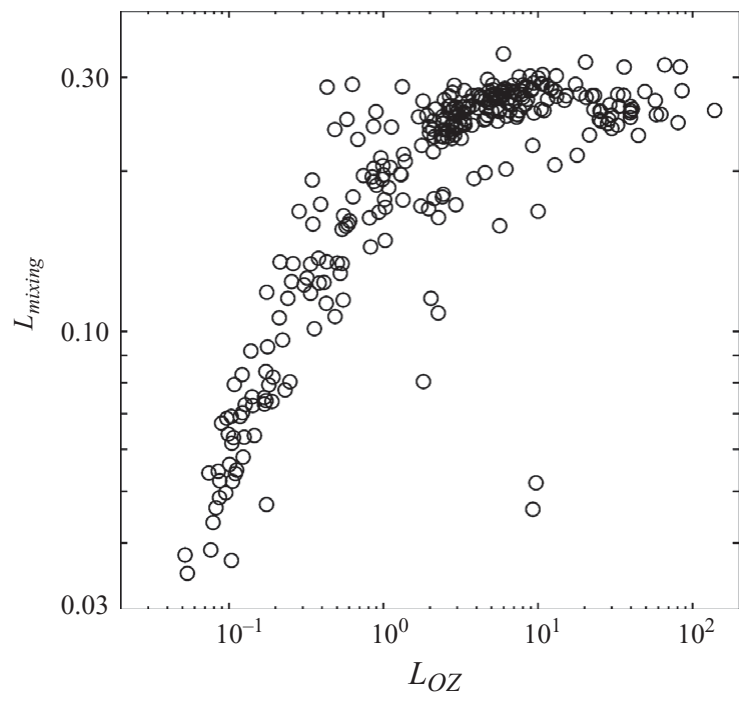

FIGURE 16. Variation of the mixing length with the Ozmidov length scale.

in its formulation; the dissipation used here was obtained from the second-order structure function which as we have illustrated is very well correlated with the energy cascade $\Pi$ (we also computed the Ozmidov scale based on the dissipation estimates from the residual $\langle\varepsilon\rangle=\langle\Pi\rangle-\left\langle B_{S G S}\right\rangle$ and the agreement was just as good, though the values of $L_{O Z}$ were lower). While further investigations of the best index of stability under stable conditions are beyond the scope of this paper, it is clear that indices relying on gradients of potential temperature seem more relevant than those relying on heat flux.

More interesting to note in these figures is the fact that the decrease in $c_{s}$ and increase in $\operatorname{Pr}$ start almost exactly at $\Delta / L_{O Z}=1$. In the present analysis, $\Delta / z>1$ and hence the filter cutoff falls in the production range, which explains the low value of $c_{s}$ at neutral stability compared to the value of 0.16 expected in the inertial subrange. Hence when $\Delta / L_{O Z}<1$, the filter cutoff is in the production range and the SGSs are affected by the proximity to the wall but not by buoyancy. For $\Delta / L_{O Z}>1$, the filter cutoff is in the buoyancy subrange and the value of $c_{s}$ decreases further; the SGSs are now affected by both the proximity to the wall and the buoyancy. We have verified that the decrease in $c_{s}$ at higher stabilities is intimately related to an increase in the strain-rate tensor (denominator of $c_{s}$ in (8.1)) rather than a decrease in the TKE cascade (numerator of $c_{s}$ in (8.1)). Similarly, an increase in the value and scatter of $\mathrm{Pr}$ is observed when $\Delta / L_{\mathrm{OZ}}$ exceeds 1 .

We now look at the mixing length scale $\left(L_{\text {mixing }}=c_{s} \Delta\right)$ implied by the coefficients above. Figure 16 depicts the variation of this scale with the Ozmidov length scale. The two length scales are very well correlated, though the relation is nonlinear. The mixing length is relatively constant at neutral conditions (high $L_{O Z}$ ) and then decreases rapidly as stability sets in (as $L_{O Z}$ decreases).

\section{Modelled fluxes}

The coefficients above are, as previously discussed, computed to match measured and modelled dissipations. Here we investigate how these coefficients perform in modelling the significant vertical fluxes presented in $\S 6$. We re-plot figure 4 after 


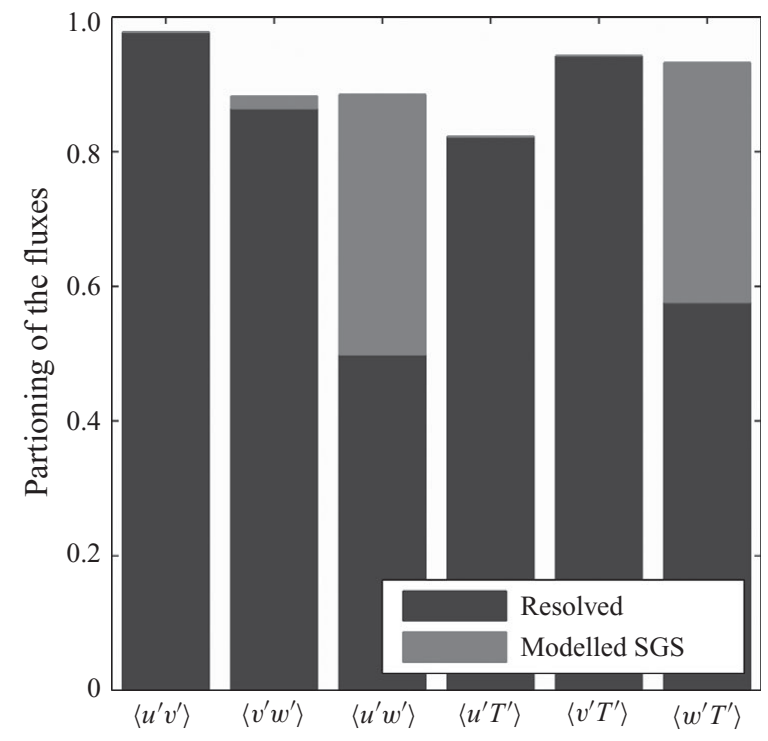

FIGURE 17. Partitioning of the fluxes between resolved and modelled SGS, as a fraction of the total Reynolds fluxes.

replacing the measured SGS fractions with the modelled SGS fractions (computed as fractions of the Reynolds stresses). The results are reported in figure 17 and indicate that the SGS model underestimates the measured SGS fluxes (the missing portion corresponds to the space between the modelled SGS bar and the top of the figure). Nevertheless, we see that for the vertical fluxes, which were the most critical, the model does a relatively good job and reproduces a significant fraction. The resolved and SGS modelled fluxes end up recapturing over $80 \%$ of the Reynolds fluxes for all components (about $90 \%$ for the vertical fluxes).

Alternatively, and in view of the importance of the SGS vertical fluxes near the wall, one can compute the model coefficients that would match the measured and modelled vertical fluxes. The resulting formulations for the 'flux-based' optimal coefficients would be

$$
c_{s, f l u x}^{2}=\frac{-\left\langle\tau_{13}\right\rangle}{2(\Delta)^{2}\left\langle|\widetilde{S}| \widetilde{S}_{13}\right\rangle}, \quad \operatorname{Pr}_{f f u x}^{-1} c_{s, f l u x}^{2}=\frac{-\left\langle q_{3}^{\text {heat }}\right\rangle}{\left\langle\Delta^{2}|\widetilde{S}|(\partial \widetilde{\theta} / \partial z)\right\rangle} .
$$

The variation of the flux-based coefficients with stability was analysed and found to be similar to the variation of the coefficients based on dissipations, albeit with different values of the coefficients. However, we are here more interested in a direct comparison of the flux-based and dissipation-based coefficients since the goal of $\S 10$ is to investigate whether the discrepancy between these coefficients increases at higher stabilities. This would indicate more challenging conditions for the SGS model in the sense that it would not be able to capture both dissipations and fluxes.

We plot the $c_{s}$ (based on dissipation) versus $c_{s, f l u x}$, with the data points coloured according to the logarithm of the stability function $\ln \left(z / L_{O Z}\right)$. The results, depicted in figure 18, show that for strong stabilities (low $c_{s}$, high and positive $\ln \left(z / L_{O Z}\right)$, red to yellow), the two coefficients follow similar trends, albeit with noticeable scatter especially in relative terms. At neutral stability (higher $c_{s}, \ln \left(z / L_{O Z}\right)<0$ corresponding to $z / L_{O Z}<1$, cyan to blue), the relative scatter is a bit lower but there is a visible 


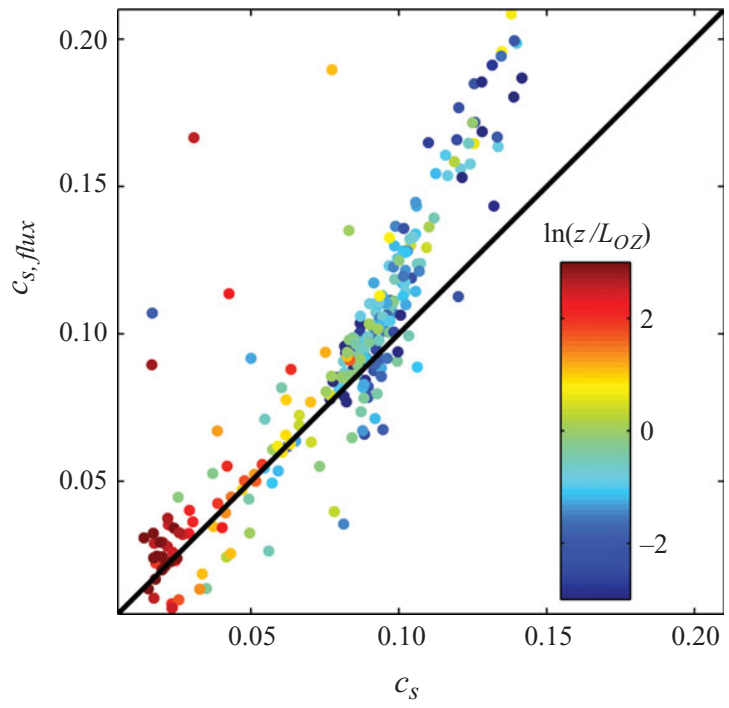

FIGURE 18. Comparison of the Smagorinsky coefficients matching measured TKE dissipation and vertical momentum flux.

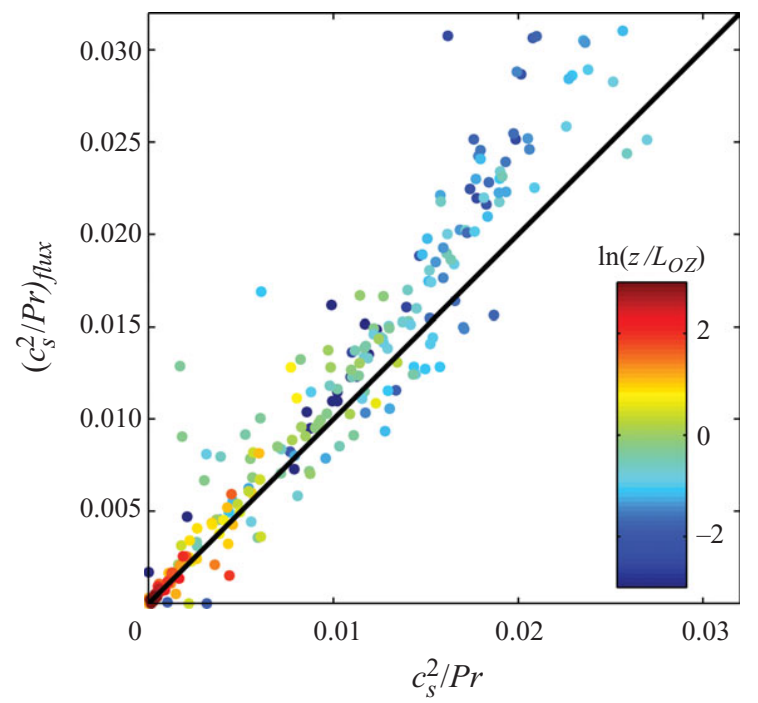

FIGURE 19. Comparison of the model coefficients matching measured temperature variance dissipation and vertical heat flux.

departure towards higher values of the flux-based coefficient, implying that on average the dissipation-based coefficient would underestimate mean fluxes. But overall, the agreement is reasonable. Similar conclusions can be drawn for the coefficient of the SGS heat model $c_{s}^{2} / \operatorname{Pr}_{S G S}$ number (figure 19). The coefficients agree better at high stabilities with less scatter than was observed for $c_{s}$; while at moderate and neutral stabilities there is a clear difference in the trend and the flux-based coefficient is again higher than the dissipation based one. The differences between the optimal coefficients based on fluxes and dissipations are to be expected in view of the misalignment of the modelled and measured stresses depicted in $\S 8$ and the model deficiencies it illustrates. 


\section{Effect of non-turbulent modes on SGS dynamics}

In the light of these results, we sought to directly assess the impact of the largescale, non-turbulent modes (waves, submeso modes, etc.) that complicate the sensing and modelling of stable ABLs on SGS dynamics. We used two mesoscale-turbulent flow separation techniques: a simple high-pass Gaussian filter and an MRD filter (Vickers \& Mahrt 2003; Nakamura \& Mahrt 2005) with filter sizes of 100 and 180 s for both techniques; the choice of these scales is based on the findings of Basu et al. (2006) and references therein and Vickers \& Mahrt (2003). With the high-pass Gaussian filter, moderate differences were observed in the values of the computed coefficients (median relative difference of $6 \%$ for $c_{s}$ and $7 \%$ for $\mathrm{Pr}$ ) between runs with no filtering at all and runs with the high-pass Gaussian filter of $100 \mathrm{~s}$. The differences were, as expected, lower for the $180 \mathrm{~s}$ high-pass filter. The Reynolds fluxes on the other hand showed higher differences since the larger, flux-carrying turbulent and non-turbulent modes are eliminated by the filtering. With the $100 \mathrm{~s}$ Gaussian filter, the median relative difference for $H$ was about $13 \%$ and for $u_{*}$ about $27 \%$, resulting in a median relative difference in $z / L_{M O}$ of about $39 \%$. Hence, differences between filtered and unfiltered plots of $\operatorname{Pr}$ or $c_{s}$ versus $z / L_{M O}$ would be mainly due to differences in $z / L_{M O}$.

In addition, the observed changes in the SGS vertical fluxes of heat and momentum were $1.5 \%$ and $3 \%$, respectively. These very small differences indicate that all our SGS quantities and coefficients are minimally affected by the pre-filtering of the data that removes mesoscale disturbances. The effect of these disturbances on the results presented in this paper would be mainly due to differences in the stability parameters and resolved flow quantities.

A similar analysis with the MRD filtering (also with a filter scale of $100 \mathrm{~s}$ ) led to the same conclusions. Median relative differences between filtered and unfiltered computations were as follows: $4 \%$ for $c_{s}, 8 \%$ for $\operatorname{Pr}, 9 \%$ for $H, 19 \%$ for $u_{*}$ and $28 \%$ for $z / L_{M O}$. Again for the vertical SGS fluxes, the differences were minor: $2 \%$ for SGS momentum flux and $1 \%$ for SGS heat flux.

This analysis strongly suggests that the SGS eddies are not interacting with scales that are larger than our separation filter scale. This partially explains why the effect of stability on SGS dynamic was found to be predictable: the SGSs feel the effect of stability simply though the SGS buoyant TKE destruction term which increases consistently with increasing stability. They do not seem to interact significantly with waves and other large stable ABL features, although the effect of these features is felt indirectly through their impact on the mean flow and the large resolved turbulent scales. These findings concur with the spectrally local energy transfer to the SGSs from the smallest resolved scales (rather than from the whole turbulence spectrum) that were reported to dominate SGS dynamics (Domaradzki, Liu \& Brachet 1993; Eyink \& Aluie 2009; Aluie \& Eyink 2009; although we note that Eyink \& Aluie show that this local transfer, in the inertial subrange, does not require non-local triadic interactions as argued by Domaradzki, Liu \& Brachet).

\section{Conclusions}

An extensive field experiment was performed over the Plaine-Morte glacier in Switzerland to investigate the role, dynamics and modelling of SGS turbulence for LES of wall-bounded flows under statically stable conditions. Two arrays with a total of 12 sonic anemometers were deployed, along with many supporting measurements. The arrays provide turbulence data which can be spatially filtered to divide them 
into a resolved component and an SGS component; a priori investigation of SGS dynamics and models could then be performed.

First we investigated the properties of the SGSs under stable conditions to determine whether they (i) are fundamentally different from unstable and neutral conditions and/or (ii) become proportionally more relevant under increasing stability. We found the fractions of the fluxes (of momentum and heat) falling in the SGS range to be high (up to $50 \%$ of total fluxes) when the filter scale is about twice the height above the ground (similar to the first grid node above the wall in many LES codes that do not resolve the viscous sublayer). The SGS fraction of the vertical fluxes was the highest, compared to the horizontal fluxes. All fractions of the SGS fluxes and TKE increased as the filter scale was increased. However, we found no noticeable trend of increasing SGS fractions as the stability increased.

We then computed the SGS cascade of TKE and temperature variance and compared them to the viscous dissipation estimates based on the second-order and third-order structure functions; the two quantities matched well over a wide range of dissipations. This was attributed to the fact that the dissipation estimates based on the structure functions are actually measures of spectral cascades rather than of dissipation-range viscous effects. The direct destruction of TKE by buoyancy was found to become important only at high stabilities. At the highest stabilities studied in this paper, the results indicated that the fractions of the TKE destroyed by buoyancy in the resolved scales, destroyed by buoyancy in the SGS scales, and viscously dissipated are on the same order of magnitude. As such, a large fraction of the TKE still has to cascade to the SGS scales before it is dissipated by viscosity or destroyed by SGS buoyancy; this cascade process hence remains the main sink of TKE (and the only sink of scalar variance) from the resolved scales.

These results for the fluxes and dissipations suggest that the role of the SGSs, while very important for both the vertical fluxes and the dissipations, does not increase in importance under stable conditions. More importantly, the function of the SGS scales under stable conditions is fundamentally similar to neutral and unstable conditions: (i) these scales still represent the main TKE sink for the resolved scales and (ii) they still carry a significant fraction of the turbulent fluxes (over $50 \%$ of the total) when the filter scale is in the production range (close to the wall in actual LES) as is observed under neutral stabilities, although unstable conditions appear to significantly reduce the SGS fractions (Porte-Agel et al. 2001a; Sullivan et al. 2003)

We computed the Smagorinsky model coefficients for momentum and heat; the coefficient values vary with stability in accordance with the findings of Kleissl et al. (2003, 2004). While considerable scatter was observed when the coefficients were plotted versus the stability parameter based on the Obukhov length scale, this scatter was not a function of stability and was shown to be due to the inadequacy of the Obukhov length to characterize stability. Better results with significantly less scatter were obtained when the Ozmidov length scale was used in the stability parameter $\left(z / L_{O Z}\right)$ instead. The Smagorinsky coefficient decreased with stability, while the SGS Prandtl number increased. This shows that imposing a fixed coefficient in LES is not adequate and that a dynamic procedure is strongly preferable. This analysis was performed to answer the following question: are model coefficients more challenging to estimate under stable conditions? The results suggest that the variation of the coefficient with stability is consistently predictable (e.g. it follows the findings of Kleissl et al. 2003 for another field experiment). In addition, the comparison of the Smagorinsky coefficients that reproduce the kinetic energy dissipation or temperature variance dissipation and the vertical momentum or heat flux suggests that their 
agreement is not highly sensitive to stability (high stabilities seem to give better trend but higher scatter). These two findings, along with the findings of Kleissl et al. (2004), indicate that the coefficients can be predicted with the same accuracy using scale-dependent dynamic models, and that the determined coefficients based on TKE dissipation will not yield higher errors in the estimates of the vertical fluxes under stable conditions, compared to neutral or convective conditions.

Furthermore, we investigated the alignment of the SGS stress and resolved strain tensors, which is assumed perfect in the Smagorinsky model, but is well known not to be so. We found the misalignment to be similar to that observed at several other sites and in laboratory square-duct flow under various stability conditions. This alignment seems to be a robust and universal characteristic of turbulence in wall-bounded flows. On the other hand, the alignment between the SGS heat flux and the resolved temperature gradients was found to be sensitive to stability in other studies (Chamecki 2010).

This partially answers our last question about whether there are inherent difficulties in modelling SGS turbulence under stable and very stable conditions similar to the difficulties we face in RANS models. What we can conclude from this analysis is that particular difficulties under stable conditions are unlikely to be related to the SGS model; whereas modelling of the full turbulence spectrum in RANS can be considerably more challenging. While SGS models remain very important for LES results under all stabilities as numerous previous studies demonstrate (Kosovic 1997; Porte-Agel et al. 2000a; Bou-Zeid et al. 2005; Beare et al. 2006; Kleissl et al. 2006; Stoll \& Porte-Agel 2006), this study suggests that the role and modelling of the SGSs under stable conditions is mainly affected by buoyant TKE destruction, in a predictable manner (e.g. decrease of $c_{s}$ ). Tests that we performed using high-pass filtering to separate the turbulent and non-turbulent modes in the flow suggested that the SGSs are not interacting directly with large mesoscale or submeso scale disturbances (waves, instabilities, etc.); this explains the relative consistency of their dynamics under varying stability.

This of course does not preclude other problems, of great significance in LES of the ABL, under stable conditions such as:

(i) The use of a wall-model at the first grid point introduces other challenges and calls for stability functions that are not always well known and need the stability parameter based on the Obukhov length scale, which as we suggested may not be well suited for stable conditions (for a broader discussion on wall modelling in LES, see e.g. Marusic, Kunkel \& Porte-Agel 2001, Piomelli \& Balaras 2002 and Chamorro \& Porte-Agel 2009).

(ii) Imposing the heat flux at the wall leads to the problem of double solutions (one heat flux could correspond to two different stabilities, depending on the friction velocity) as discussed, e.g. in Basu et al. (2008).

(iii) Characteristic large-scale eddies in a stable flow are smaller than under neutral and unstable conditions (e.g. in the stable ABL, large eddies might not exceed $100 \mathrm{~m}$, compared with scales of up to $1000 \mathrm{~m}$ under unstable conditions). In addition, stable ABLs can exhibit thin regions of high shear and turbulence production (such as low-level jets) that might also be poorly resolved (Mahrt 1998) and where the filter scale might lie in the production range (as opposed to neutral and unstable ABLs, where this is only the case near the wall). As such, stable ABL simulations might be more sensitive to the grid resolution and the ability of the simulation to properly capture the resolved, large-scale flow structures.

(iv) Under unstable or neutral conditions, the flow is generally dominated by turbulence generated in the atmospheric surface layer, which is contained within the LES domain. Under stable conditions, turbulence is less dominant and could 


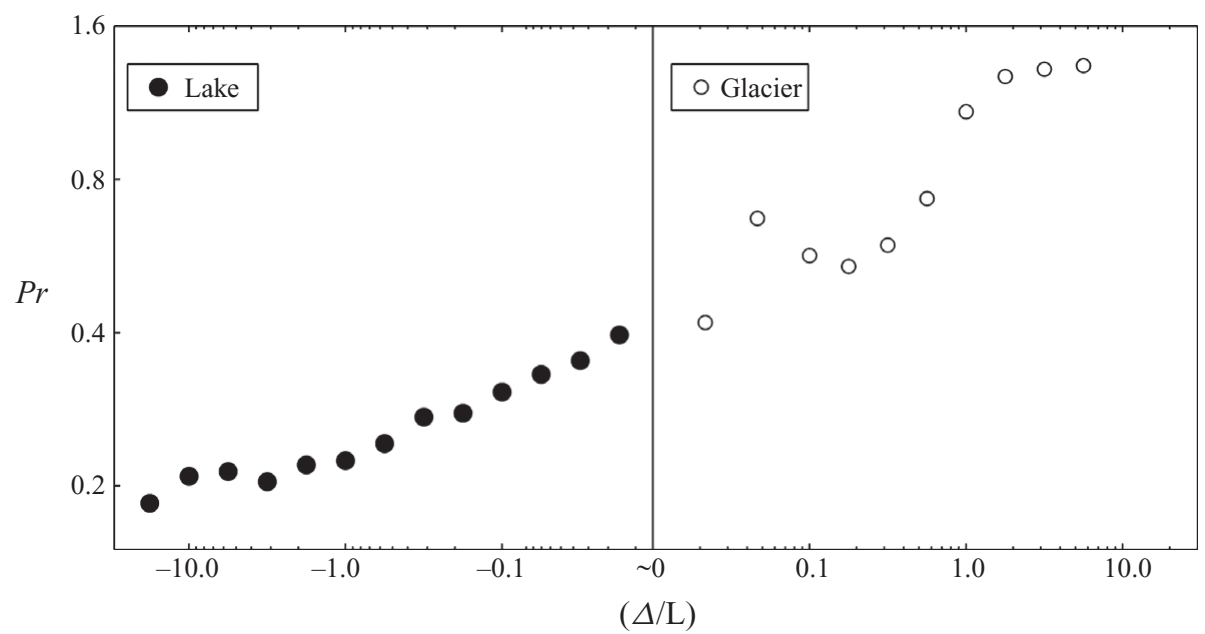

FIGURE 20. Variation of the SGS Prandtl number for unstable and stable conditions (note that both axes are in logarithmic scale).

be generated by breaking waves or regions of high shear above the ABL (outside of the LES domain) and propagating down into the stable ABL (Mahrt 1999). When LES and experimental data are compared, the effects of atmospheric dynamics occurring during the experiment, but laying outside of the simulation domain, are difficult to account for. Therefore, for comparison studies, it is preferable to focus on experimental periods where such features are not present.

To conclude, the results of the present analysis show that SGS modelling in LES of wall-bounded flows under stable conditions shares many of the properties encountered under neutral and unstable conditions. However, other aspects of LES under stable conditions (points (i)-(iv) above) remain challenging and further investigations that focus on these aspects are needed.

The authors would like to thank the Swiss National Science Foundation for its support for this work through grant number 200021-107910 and through the National Competence Center in Research on Mobile Information and Communication Systems (NCCR-MICS) under grant number 5005-67322. E.B.-Z. is also supported through the Princeton Environmental Institute's Energy Grand Challenges Program. C.M. is supported by the US National Science Foundation under grant EAR-0609690. Ms N. Vercauteren computed the SGS Prandtl numbers from the data over the lake, reported in Vercauteren et al. (2008). We would like to thank Dr D. Vickers for sharing his MRD subroutines with us. We are also grateful to our colleagues at the Crans-Montana Ski area for their assistance in the field deployment and to the many colleagues at the Environmental Fluid Mechanics and Hydrology Laboratory of EPFL, without whom this Alpine field experiment would not have been possible.

\section{Appendix}

Figure 13 depicted the increase of $\mathrm{Pr}$ as the stability increased. In a similar analysis performed by Vercauteren et al. (2008) for unstable atmospheric conditions, the SGS Prandtl number was found to decrease as the stability parameter decreased to higher negative values (increased in magnitude). The data from SnoHATS and from the lake-atmosphere turbulent exchanges experiment analysed by Vercauteren et al. (2008) are depicted together in figure 20. We see a clear increase in $\operatorname{Pr}$ as 
the atmospheric stability changes from highly unstable $\left(\operatorname{Pr} \approx 0.2\right.$ at $\left.\Delta / L_{M O} \approx-10\right)$ to neutral $\left(\operatorname{Pr} \approx 0.4\right.$ at $\left.\Delta / L_{M O} \approx 0\right)$ to stable $\left(\operatorname{Pr} \approx 1.4\right.$ at $\left.\Delta / L_{M O} \approx 10\right)$. Except near neutral stabilities, where the temperature variations are small and their relative measurement errors (and that of heat fluxes needed in $L_{M O}$ ) are large, the trend is clear and robust over a large range of stabilities and indicates that buoyancy plays a significant role in modulating the structure of turbulence and the efficiency of turbulent transport, even at the small SGSs. As such, buoyant TKE production (unstable) seems to increase the efficiency of heat transport compared to momentum transport, while buoyant TKE destruction (stable) tends to reduce the efficiency of heat transport relative to momentum transport.

\section{REFERENCES}

Aluie, H. \& EyinK, G. L. 2009 Localness of energy cascade in hydrodynamic turbulence. Part II. Sharp spectral filter. Phys. Fluids 21 (11), 115108.

Armenio, V. \& Sarkar, S. 2002 An investigation of stably stratified turbulent channel flow using large-eddy simulation. J. Fluid Mech. 459, 1-42.

Ashurst, W. T., Kerstein, A. R., Kerr, R. M. \& Gibson, C. H. 1987 Alignment of vorticity and scalar gradient with strain rate in simulated Navier-Stokes turbulence. Phys. Fluids 30 (8), 2343-2353.

Basu, S., Holtslag, A. A. M., Van De Wiel, B. J. H., Moene, A. F. \& Steeneveld, G. J. 2008 An inconvenient 'truth' about using sensible heat flux as a surface boundary condition in models under stably stratified regimes. Acta Geophys. 56 (1), 88-99.

Basu, S., Porte-Agel, F., Foufoula-Georgiou, E., Vinuesa, J. F. \& Pahlow, M. 2006 Revisiting the local scaling hypothesis in stably stratified atmospheric boundary-layer turbulence: an integration of field and laboratory measurements with large-eddy simulations. Boundary-Layer Meteorol. 119 (3), 473-500.

Beare, R. J. \& Macvean, M. K. 2004 Resolution sensitivity and scaling of large-eddy simulations of the stable boundary layer. Boundary-Layer Meteorol. 112 (2), 257-281.

Beare, R. J., MacVean, M. K., Holtslag, A. A. M., Cuxart, J., Esau, I., Golaz, J. C., Jimenez, M. A., Khairoutdinov, M., Kosovic, B., Lewellen, D., Lund, T. S., Lundquist, J. K., McCabe, A., Moene, A. F., Noh, Y., Raasch, S. \& Sullivan, P. 2006 An intercomparison of large-eddy simulations of the stable boundary layer. Boundary-Layer Meteorol. 118 (2), 247-272.

BeljaARs, A. C. M. 1995 The impact of some aspects of the boundary layer scheme in the ECMWF model. In Seminar on Parametrization of Subgrid Scale Physical Processes, 5-9 September 1994 - ECMWF, Reading, UK, pp. 125-161.

Bou-Zeid, E., Meneveau, C. \& Parlange, M. B. 2004 Large-eddy simulation of neutral atmospheric boundary layer flow over heterogeneous surfaces: Blending height and effective surface roughness. Water Resour. Res. 40 (2), W02505.

Bou-Zeid, E., Meneveau, C. \& Parlange, M. B. 2005 A scale-dependent Lagrangian dynamic model for large eddy simulation of complex turbulent flows. Phys. Fluids 17 (2), 025105.

Bou-Zeid, E., Parlange, M. B. \& Meneveau, C. 2007 On the parameterization of surface roughness at regional scales. J. Atmos. Sci. 64 (1), 216-227.

Bou-Zeid, E., Vercauteren, N., Parlange, M. B. \& Meneveau, C. 2008 Scale dependence of subgrid-scale model coefficients: an a priori study. Phys. Fluids 20 (11), $115106-115106$.

Brasseur, J. G. \& WeI, T. 2010 Designing large-eddy simulation of the turbulent boundary layer to capture law-of-the-wall scaling. Phys. Fluids 22 (2), 021303.

De Bruin, H. A. R. 1994 Analytic solutions of the equations governing the temperature fluctuation method. Boundary-Layer Meteorol. 68 (4), 427-432.

BRUTSAERT, W. 1998 Land-surface water vapor and sensible heat flux: spatial variability, homogeneity and measurement scales. Water Resour. Res. 34 (10), 2433-2442.

Canuto, V. M. \& MinotTi, F. 1993 Stratified turbulence in the atmosphere and oceans - a new subgrid model. J. Atmos. Sci. 50 (13), 1925-1935. 
Chamecki, M. 2010 Modelling subgrid-scale heat fluxes in the neutral and stratified atmospheric boundary layer. J. Turbul. 11 (13), 1-16.

Chamecki, M. \& Dias, N. L. 2004 The local isotropy hypothesis and the turbulent kinetic energy dissipation rate in the atmospheric surface layer. Q. J. R. Meteorol. Soc. 130 (603), 2733-2752.

Chamecki, M., Meneveau, C. \& Parlange, M. B. 2007 The local structure of atmospheric turbulence and its effect on the Smagorinsky model for large eddy simulation. J. Atmos. Sci. 64 (6), 19411958.

Chamorro, L. P. \& Porte-Agel, F. 2009 Velocity and surface shear stress distributions behind a rough-to-smooth surface transition: a simple new model. Boundary-Layer Meteorol. 130 (1), $29-41$.

Champagne, F. H., Friehe, C. A., Larue, J. C. \& Wyngaard, J. C. 1977 Flux measurements, flux estimation techniques and fine-scale turbulence measurements in unstable surface layer over land. J. Atmos. Sci. 34 (3), 515-530.

Cheng, Y. G. \& Brutsaert, W. 2005 Flux-profile relationships for wind speed and temperature in the stable atmospheric boundary layer. Boundary-Layer Meteorol. 114 (3), 519-538.

Cheng, Y. G., Parlange, M. B. \& Brutsaert, W. 2005 Pathology of Monin-Obukhov similarity in the stable boundary layer. J. Geophys. Res. Atmos. 110 (D6), D06101.

Chimonas, G. 1999 Steps, waves and turbulence in the stably stratified planetary boundary layer. Boundary-Layer Meteorol. 90 (3), 397-421.

Cui, G. X., Zhou, H. B., Zhang, Z. S. \& Shao, L. 2004 A new dynamic subgrid eddy viscosity model with application to turbulent channel flow. Phys. Fluids 16 (8), 2835-2842.

Dalaudier, F. \& Sidi, C. 1990 Some characteristics of the turbulent buoyancy subrange. Adv. Space Res. 10 (10), 37-40.

DEARDORFF, J. W. 1980 Stratocumulus-capped mixed layers derived from a 3-dimensional model. Boundary-Layer Meteorol. 18 (4), 495-527.

Derbyshire, S. H. 1999 Stable boundary-layer modelling: established approaches and beyond. Boundary-Layer Meteorol. 90 (3), 423-446.

Domaradzki, J. A., Liu, W. \& Brachet, M. E. 1993 An analysis of subgrid-scale interactions in numerically simulated isotropic turbulence. Phys. Fluids A 5 (7), 1747-1759.

Dubrulle, B., Laval, J. P., Sullivan, P. P. \& Werne, J. 2002 A new dynamical subgrid model for the planetary surface layer. Part I: The model and a priori tests. J. Atmos. Sci. 59 (4), 861-876.

Eyink, G. L. \& Aluie, H. 2009 Localness of energy cascade in hydrodynamic turbulence. Part I. Smooth coarse graining. Phys. Fluids 21 (11), 115107.

Forrer, J. \& Rotach, M. W. 1997 On the turbulence structure in the stable boundary layer over the Greenland ice sheet. Boundary-Layer Meteorol. 85 (1), 111-136.

Germano, M. 1986 A proposal for a redefinition of the turbulent stresses in the filtered NavierStokes equations. Phys. Fluids 29 (7), 2323-2324.

Germano, M., Piomelli, U., Moin, P. \& Cавот, W. H. 1991 A dynamic subgrid-scale eddy viscosity model. Phys. Fluids A 3 (7), 1760-1765.

Gullbrand, J. \& ChOw, F. K. 2003 The effect of numerical errors and turbulence models in large-eddy simulations of channel flow, with and without explicit filtering. J. Fluid Mech. 495, 323-341.

Guo, Z. C., Dirmeyer, P. A., Koster, R. D., Bonan, G., Chan, E., Cox, P., Gordon, C. T., Kanae, S., Kowalczyk, E., Lawrence, D., Liu, P., Lu, C. H., Malyshev, S., McAvaney, B., McGregor, J. L., Mitchell, K., Mocko, D., Oki, T., Oleson, K. W., Pitman, A., Sud, Y. C., Taylor, C. M., Verseghy, D., Vasic, R., Xue, Y. K. \& Yamada, T. 2006 GlaCE: the global land-atmosphere coupling experiment. Part II: Analysis. J. Hydrometeorol. 7 (4), 611-625.

HAN, Z. W., UEDA, H. \& AN, J. L. 2008 Evaluation and intercomparison of meteorological predictions by five MM5-PBL parameterizations in combination with three land-surface models. Atmos. Environ. 42 (2), 233-249.

Handorf, D., Foken, T. \& Kottmeier, C. 1999 The stable atmospheric boundary layer over an Antarctic ice sheet. Boundary-Layer Meteorol. 91 (2), 165-189.

Higgins, C. W., Meneveau, C. \& Parlange, M. B. 2007 The effect of filter dimension on the subgrid-scale stress, heat flux and tensor alignments in the atmospheric surface layer. $J$. Atmos. Ocean. Technol. 24 (3), 360-375. 
Higgins, C. W., Meneveau, C. \& Parlange, M. B. 2009 Geometric alignments of the subgrid-scale force in the atmospheric boundary layer. Boundary-Layer Meteorol. 132 (1), 1-9.

Higgins, C. W., Parlange, M. B. \& Meneveau, C. 2003 Alignment trends of velocity gradients and subgrid-scale fluxes in the turbulent atmospheric boundary layer. Boundary-Layer Meteorol. 109 (1), 59-83.

Horst, T. W., Kleissl, J., Lenschow, D. H., et al. 2004 HATS: field observations to obtain spatially filtered turbulence fields from crosswind arrays of sonic anemometers in the atmospheric surface layer. J. Atmos. Sci. 61 (13), 1566-1581.

Kaimal, J. C. \& Finnigan, J. J. 1994 Atmospheric Boundary Layer Flows: Their Structure and Measurement. Oxford University Press.

Kaimal, J. C., Izumi, Y., WyngaARd, J. C. \& Cote, R. 1972 Spectral characteristics of surface-layer turbulence. Q. J. R. Meteorol. Soc. 98 (417), 563-589.

Katul, G., Hsieh, C. I. \& Sigmon, J. 1997 Energy-inertial scale interactions for velocity and temperature in the unstable atmospheric surface layer. Boundary-Layer Meteorol. 82 (1), 49-80.

King, J. C., Connolley, W. M. \& Derbyshire, S. H. 2001 Sensitivity of modelled Antarctic climate to surface and boundary-layer flux parametrizations. Q. J. R. Meteorol. Soc. 127 (573), 779-794.

Kleissl, J., Kumar, V., Meneveau, C. \& Parlange, M. B. 2006 Numerical study of dynamic Smagorinsky models in large-eddy simulation of the atmospheric boundary layer: validation in stable and unstable conditions. Water Resour. Res. 42 (6), W06D10.

Kleissl, J., Meneveau, C. \& Parlange, M. B. 2003 On the magnitude and variability of subgridscale eddy-diffusion coefficients in the atmospheric surface layer. J. Atmos. Sci. 60 (19), 2372-2388.

Kleissl, J., Parlange, M. B. \& Meneveau, C. 2004 Field experimental study of dynamic Smagorinsky models in the atmospheric surface layer. J. Atmos. Sci. 61 (18), 2296-2307.

Kosovic, B. 1997 Subgrid-scale modelling for the large-eddy simulation of high-Reynolds-number boundary layers. J. Fluid Mech. 336, 151-182.

Kosovic, B. \& Curry, J. A. 2000 A large eddy simulation study of a quasi-steady, stably stratified atmospheric boundary layer. J. Atmos. Sci. 57 (8), 1052-1068.

Koster, R. D., Guo, Z. C., Dirmeyer, P. A., Bonan, G., Chan, E., Cox, P., Davies, H., Gordon, C. T., Kanae, S., Kowalczyk, E., Lawrence, D., Liu, P., Lu, C. H., Malyshev, S., McAvaney, B., Mitchell, K., Mocko, D., Oki, T., Oleson, K. W., Pitman, A., Sud, Y. C., Taylor, C. M., Verseghy, D., Vasic, R., Xue, Y. K. \& Yamada, T. 2006 GLACE: the global land-atmosphere coupling experiment. Part I: Overview. J. Hydrometeorol. 7 (4), 590-610.

Kumar, V., Kleissl, J., Meneveau, C. \& Parlange, M. B. 2006 Large-eddy simulation of a diurnal cycle of the atmospheric boundary layer: atmospheric stability and scaling issues. Water Resour. Res. 42 (6), W06D09.

Kunkel, G. J. \& Marusic, I. 2006 Study of the near-wall-turbulent region of the high-Reynoldsnumber boundary layer using an atmospheric flow. J. Fluid Mech. 548, 375-402.

LILLY, D. K. 1967 The representation of small scale turbulence in numerical simulation experiments. In IBM Scientific Computing Symposium on Environmental Sciences, White Plains, NY, pp. 195-209.

MAhrt, L. 1998 Stratified atmospheric boundary layers and breakdown of models. Theor. Comput. Fluid Dyn. 11 (3-4), 263-279.

MAHRT, L. 1999 Stratified atmospheric boundary layers. Boundary-Layer Meteorol. 90 (3), 375-396.

MALHI, Y. S. 1995 The significance of the dual solutions for heat fluxes measured by the temperature fluctuation method in stable conditions. Boundary-Layer Meteorol. 74 (4), 389-396.

Marusic, I., Kunkel, G. J. \& Porte-Agel, F. 2001 Experimental study of wall boundary conditions for large-eddy simulation. J. Fluid Mech. 446, 309-320.

Mason, P. J. 1989 Large-eddy simulation of the convective atmospheric boundary layer. J. Atmos. Sci. 46 (11), 1492-1516.

Meneveau, C. 1994 Statistics of turbulence subgrid-scale stresses - necessary conditions and experimental tests. Phys. Fluids 6 (2), 815-833.

Meneveau, C. \& Katz, J. 2000 Scale-invariance and turbulence models for large-eddy simulation. Annu. Rev. Fluid Mech. 32, 1-32. 
Metais, O. \& Herring, J. R. 1989 Numerical simulations of freely evolving turbulence in stably stratified fluids. J. Fluid Mech. 202, 117-148.

Mirocha, J. D., Kosovic, B. \& Curry, J. A. 2005 Vertical heat transfer in the lower atmosphere over the Arctic Ocean during clear-sky periods. Boundary-Layer Meteorol. 117 (1), 37-71.

Moeng, C. H. 1984 A large-eddy-simulation model for the study of planetary boundary-layer turbulence. J. Atmos. Sci. 41 (13), 2052-2062.

NAKamuRA, R. \& MahrT, L. 2005 A study of intermittent turbulence with cases-99 tower measurements. Boundary-Layer Meteorol. 114 (2), 367-387.

Pahlow, M., Parlange, M. B. \& Porte-Agel, F. 2001 On Monin-Obukhov similarity in the stable atmospheric boundary layer. Boundary-Layer Meteorol. 99 (2), 225-248.

Piomelli, U. \& Balaras, E. 2002 Wall-layer models for large-eddy simulations. Annu. Rev. Fluid Mech. 34, 349-374.

Pope, S. B. 2000 Turbulent Flows. Cambridge University Press.

Porte-Agel, F. 2004 A scale-dependent dynamic model for scalar transport in large-eddy simulations of the atmospheric boundary layer. Boundary-Layer Meteorol. 112 (1), 81-105.

Porte-Agel, F., Meneveau, C. \& Parlange, M. B. $2000 a$ A scale-dependent dynamic model for large-eddy simulation: application to a neutral atmospheric boundary layer. J. Fluid Mech. 415, 261-284.

Porte-Agel, F., Pahlow, M., Meneveau, C. \& Parlange, M. B. 2001 a Atmospheric stability effect on subgrid-scale physics for large-eddy simulation. Adv. Water Resour. 24 (9-10), $1085-1102$.

Porte-Agel, F., Parlange, M. B., Meneveau, C. \& Eichinger, W. E. $2001 b$ A priori field study of the subgrid-scale heat fluxes and dissipation in the atmospheric surface layer. J. Atmos. Sci. 58 (18), 2673-2698.

Porte-Agel, F., Parlange, M. B., Meneveau, C., Eichinger, W. E. \& Pahlow, M. $2000 b$ Subgrid scale dissipation in the atmospheric surface layer: effects of stability and filter dimension. $J$. Hydrol. 1 (1), 75-87.

RANDALL, D. A. 1984 Buoyant production and consumption of turbulence kinetic-energy in cloudtopped mixed layers. J. Atmos. Sci. 41 (3), 402-413.

Sagaut, P. 2006 Large Eddy Simulation for Incompressible Flows : An Introduction. Springer.

SMAGORINSKY, J. 1963 General circulation experiments with the primitive equations. Part I. the basic experiment. Mon. Weath. Rev. 91, 99-164.

Steeneveld, G. J., Van de Wiel, B. J. H. \& Holtslag, A. A. M. 2006 Modelling the arctic stable boundary layer and its coupling to the surface. Boundary-Layer Meteorol. 118 (2), 357-378.

Stevens, B., Moeng, C. H. \& Sullivan, P. P. 1999 Large-eddy simulations-of radiatively driven convection: sensitivities to the representation of small scales. J. Atmos. Sci. 56 (23), 3963-3984.

Stoll, R. \& Porte-Agel, F. 2006 Dynamic subgrid-scale models for momentum and scalar fluxes in large-eddy simulations of neutrally stratified atmospheric boundary layers over heterogeneous terrain. Water Resour. Res. 42 (1), W01409.

Stoll, R. \& Porte-Agel, F. 2008 Large-eddy simulation of the stable atmospheric boundary layer using dynamic models with different averaging schemes. Boundary-Layer Meteorol. 126 (1), $1-28$.

Sullivan, P. P., Horst, T. W., Lenschow, D. H., Moeng, C. H. \& Weil, J. C. 2003 Structure of subfilter-scale fluxes in the atmospheric surface layer with application to large-eddy simulation modelling. J. Fluid Mech. 482, 101-139.

Sullivan, P. P., McWilliams, J. C. \& Moeng, C. H. 1994 A subgrid-scale model for largeeddy simulation of planetary boundary-layer flows. Boundary-Layer Meteorol. 71 (3), 247-276.

TaO, B., Katz, J. \& Meneveau, C. 2002 Statistical geometry of subgrid-scale stresses determined from holographic particle image velocimetry measurements. J. Fluid Mech. 457, 35-78.

Tong, C. N., Wyngaard, J. C. \& Brasseur, J. G. 1999 Experimental study of the subgrid-scale stresses in the atmospheric surface layer. J Atmos. Sci. 56 (14), 2277-2292.

Tsinober, A., Kit, E. \& Dracos, T. 1992 Experimental investigation of the field of velocity gradients in turbulent flows. J. Fluid Mech. 242, 169-192.

Venayagamoorthy, S. K. \& Stretch, D. D. 2010 On the turbulent Prandtl number in homogeneous stably stratified turbulence. J. Fluid Mech. 644, 359-369. 
Vercauteren, N., Bou-Zeid, E., Parlange, M. B., Lemmin, U., Huwald, H., Selker, J. \& Meneveau, C. 2008 Subgrid-scale dynamics of water vapour, heat and momentum over a lake. Boundary-Layer Meteorol. 128 (2), 205-228.

Vickers, D. \& MAHrT, L. 1997 Quality control and flux sampling problems for tower and aircraft data. J. Atmos. Ocean. Technol. 14 (3), 512-526.

Vickers, D. \& MAhrt, L. 2003 The co-spectral gap and turbulent flux calculations. J. Atmos. Ocean. Technol. 20 (5), 660-672.

Willis, G. E. \& Deardorff, J. W. 1976 Use of Taylors translation hypothesis for diffusion in mixed layer. Q. J. R. Meteorol. Soc. 102 (434), 817-822.

Wong, V. C. \& Lilly, D. K. 1994 A comparison of 2 dynamic subgrid closure methods for turbulent thermal convection. Phys. Fluids 6 (2), 1016-1023.

WyngaArd, J. C. 1992 Atmospheric turbulence. Annu. Rev. Fluid Mech. 24, 205-233.

Zhang, J., TAO, B. \& KaTZ, J. 1997 Turbulent flow measurement in a square duct with hybrid holographic PIV. Exp. Fluids 23 (5), 373-381. 